\title{
The influence of an interfacial hBN layer on the fluorescence of an organic molecule
}

\author{
Christine Brülke, Oliver Bauer and Moritz M. Sokolowski*
}

\author{
Full Research Paper \\ Address: \\ Institut für Physikalische und Theoretische Chemie, Universität Bonn, \\ Wegelerstr. 12, 53115 Bonn \\ Email: \\ Moritz M. Sokolowski - sokolowski@pc.uni-bonn.de \\ * Corresponding author \\ Keywords: \\ decoupling; fluorescence; hexagonal boron nitride; 3,4,9,10-perylene \\ tetracarboxylic dianhydride (PTCDA); Raman spectroscopy \\ Beilstein J. Nanotechnol. 2020, 11, 1663-1684. \\ https://doi.org/10.3762/bjnano.11.149 \\ Received: 29 May 2020 \\ Accepted: 09 October 2020 \\ Published: 03 November 2020 \\ This article is part of the thematic issue "Molecular assemblies on \\ surfaces - towards physical and electronic decoupling of organic \\ molecules". \\ Guest Editor: M. Stöhr \\ (C) 2020 Brülke et al.; licensee Beilstein-Institut. \\ License and terms: see end of document.
}

\begin{abstract}
We investigated the ability of a single layer of hexagonal boron nitride $(\mathrm{hBN})$ to decouple the excited state of the organic molecule 3,4,9,10-perylene tetracarboxylic dianhydride (PTCDA) from the supporting $\mathrm{Cu}(111)$ surface by Raman and fluorescence (FL) spectroscopy. The Raman fingerprint-type spectrum of PTCDA served as a monitor for the presence of molecules on the surface. Several broad and weak FL lines between 18,150 and $18,450 \mathrm{~cm}^{-1}$ can be detected, already from the first monolayer onward. In contrast, FL from PTCDA on a bare $\mathrm{Cu}(111)$ surface is present only from the second PTCDA layer onward. Hence, a single layer of hBN decouples PTCDA from the metal substrate to an extent that a weak radiative FL decay of the optical excitation can occur. The different FL lines can be ascribed to different environments of the adsorption sites, namely molecules adsorbed at surface defects, in large ordered domains, and located in the second layer.
\end{abstract}

\section{Introduction}

In recent years, two-dimensional materials (2DMs) have been established as a highly interesting field of studies, both in regard to their fundamental physical properties as well as their potential for technical applications [1]. Specifically, the use of 2DMs in layered heterostructures has been promoted [2,3]. Here, one challenge lies in the understanding of not only the processes in the individual materials, but also of those that occur at the interfaces between layers of different materials.
Advantageously, some 2DMs can be grown directly on metal substrates by chemical vapor deposition [2]. This is, for example, exploited when a 2DM interfacial layer is inserted between the metallic electrode and a functional organic layer of an organic electronic device, such as an organic light emitting diode [3]. The purpose of the interfacial layer is to achieve a separation or "decoupling" of the two adjacent layers. Here, the term decoupling refers to the spatial separation of the electronic 
states of the molecules and those of the underlying metal, which leads to unperturbed molecular properties [4]. A scientifically relevant question is to which extent decoupling of the organic molecules from a metal electrode is achieved when a 2DM layer in the limit of a single interfacial layer, for example, a monolayer of hexagonal boron nitride (hBN), is used.

Such a decoupling is achieved when the wave functions of the metal are spatially separated from those of the molecule leading to a reduced overlap. The overlap of molecular and metallic wave functions has, in particular, an impact on excited molecular states. For fluorescent molecules, this is observed as a strongly reduced quantum yield of the fluorescence (FL) due to non-radiant decay processes via the metal states. This phenomenon is generally referred to as "quenching" [5]. When the decoupling is not efficient, a fast and non-radiative decay of the excitation of the molecule via the metallic states prevails. Therefore, the probing of the FL of a molecule located at the outer surface of a 2DM layer grown on a metal characterizes the degree of electronic coupling of the molecular and metallic states.

For completeness, we note that quenching of an electronic excitation of a molecule in the first layers on a metal surface can be the result of interfacial charge transfer (CT) [5] or of energy transfer [6]. Here, CT is in our focus since energy transfer, although additionally present, varies less abruptly on the scale of single layers [7]. A simple energy diagram of the CT process of a fluorescent molecule across interfaces is depicted in Figure 1. As an example, we use the sample system of this work, namely, 3,4,9,10-perylene tetracarboxylic dianhydride (PTCDA) on a layer of $\mathrm{hBN}$ on $\mathrm{Cu}(111)$. Here, we consider an $\mathrm{S}_{1}$ excitation which involves mainly a HOMO/LUMO (highest occupied and lowest unoccupied molecular orbital) electronic excitation. Rapid CT leads to a delocalization of the excited electron from the LUMO into unoccupied metallic states and/or a filling of the HOMO by an electron from the metal. We note that the HOMO and LUMO for the chemisorbed molecule differ from those of the gas phase molecule. Thus, the LUMO that is drawn in Figure 1a is not identical to the LUMO in the gas phase. For a second molecular layer, even without significant overlap of the wave functions of metal and molecule, quenching is also possible, because the CT may occur from the second layer to the metal via states in the first layer [5].

In principle, the hybridization of molecular and metallic states and the dynamical CT in the excited state can be probed by photoemission spectroscopy (PES). In particular, core hole clock spectroscopy has been used to measure the time constant of dynamical CT in the valence band states as a function of the lifetime of the core hole, which is typically of the order of $10^{-14} \mathrm{~s}[8,9]$. However, this technique is insensitive to a CT process of a considerably longer time constant. A technique for detecting CT processes with a longer time constant is FL spectroscopy, in which the $\mathrm{CT}$ process competes with the radiative fluorescent decay of the excitation. It constitutes an alternative and non-radiative decay channel for the excited state. Typically, the FL lifetime of the excited state $S_{1}$ is of the order of ca. $10^{-9} \mathrm{~s}$ [5]. Hence, a CT process with a time constant that is not significantly larger will reduce the FL yield (see Figure 1). Thus, even a very small overlap of states becomes evident in the experiment as it causes a reduction of the FL yield (i.e., quenching). The degree of quenching may vary and, hence, lead to different branching ratios between the radiative and nonradiative channels. In order to obtain high FL yields of molecules on metallic substrates, thin interfacial films of consider-

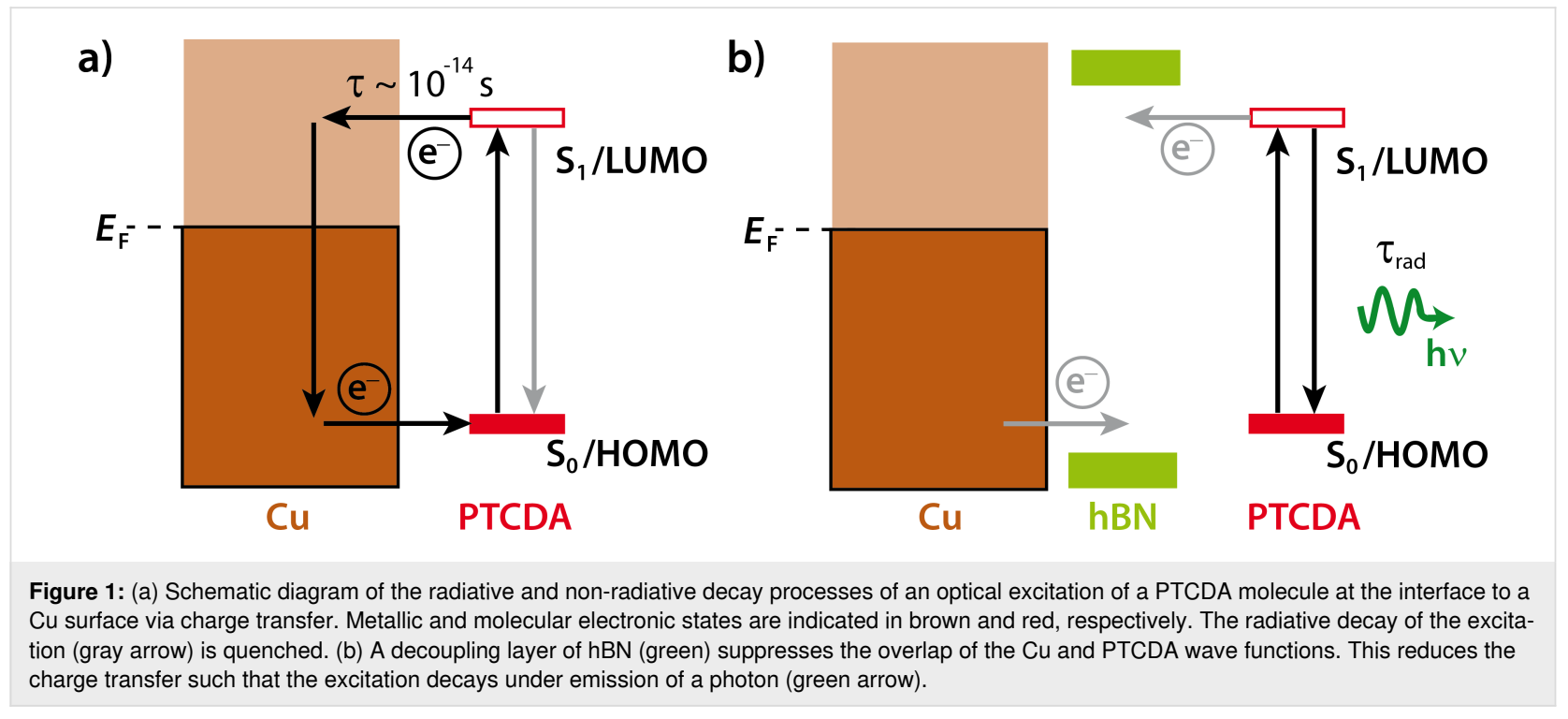


able thickness (5-10 monolayers) of alkali halides have been used in our lab [10]. In contrast, experiments on the light emission from molecules induced by scanning tunneling microscopy (STM-LE) required thin alkali halide films of two monolayers thickness in order to support tunneling [11-13].

A single layer or films of $\mathrm{hBN}$ are attractive for decoupling a molecule from an underlying metal substrate as $\mathrm{hBN}$ exhibits a wide bandgap of $5.9 \mathrm{eV}$ [14]. Perspectively, it could also provide a substrate for STM-LE experiments. Furthermore, it is of interest due to its mechanical [15], chemical [16], and thermal [17] stability, the easy synthesis of hBN monolayers on $\mathrm{Cu}$ foils for usage in devices [18], and, finally, the wide structural variety of hBN monolayers depending on the underlying metal substrate [19].

To investigate the decoupling of an organic molecule from a metal substrate by a monolayer of hBN we chose PTCDA and the $\mathrm{Cu}(111)$ surface. PTCDA serves as a planar model molecule for investigations of organic layers on surfaces [20-23]. Monolayers of $\mathrm{hBN}$ on $\mathrm{Cu}(111)$ have been investigated by several groups and are, hence, rather well understood [24-29]. In particular, we found that $\mathrm{hBN}$ on $\mathrm{Cu}(111)$ forms a flat layer at a relatively large vertical distance from the $\mathrm{Cu}(111)$ top layer of $3.24 \AA$ using an X-ray standing-waves analysis [30]. This large distance is, in principle, in agreement with the results reported independently by Schwarz and co-workers [31]. Large distances of the molecule with respect to the $\mathrm{hBN}$ and the metal interface are expected to be beneficial for the decoupling because the metal wave functions decrease exponentially into the vacuum.

We have previously shown that the bonding situation between PTCDA and $\mathrm{hBN} / \mathrm{Cu}(111)$ is weak and physisorptive [32] as opposed to the chemisorptive bond between PTCDA and $\mathrm{Cu}(111)$ [33]. Ultraviolet photoelectron spectroscopy (UPS) experiments showed that on the $\mathrm{Cu}(111)$ surface the chemical bonding leads to a filling of the LUMO [33]. In contrast, on $\mathrm{hBN} / \mathrm{Cu}(111)$, the differential energies of the PTCDA orbitals remain unaltered in comparison to those of PTCDA in the gas phase, which points to a more physisorptive bonding to the $\mathrm{hBN} / \mathrm{Cu}(111)$ surface [32]. The HOMO of PTCDA is found at ca. $2.6 \mathrm{eV}$ [32]. Hence, we can expect that both the LUMO and the HOMO are placed within the bandgap of $\mathrm{hBN}$, as indicated in Figure $1 b$. This is in agreement with the findings by Martínez-Galera et al. for PTCDA/hBN/Rh(110) [34]. From scanning tunneling spectroscopy (STS) experiments, the authors concluded that the coupling is only weak. They deduced further that the CT (in the ground state) between molecule and substrate, if present at all, is small. These differences between $\mathrm{PTCDA} / \mathrm{hBN} / \mathrm{Cu}(111)$ and PTCDA/Cu(111) are also mirrored by their vertical molecular structures. The hybridization of the electronic states of PTCDA and $\mathrm{Cu}$ leads to a distortion of the molecule where the oxygen atoms are pushed away from the substrate and out of the molecular plane [35]. On $\mathrm{hBN} / \mathrm{Cu}(111)$, the molecule remains essentially flat and at a very large vertical distance from the hBN layer of $3.37 \AA$ [32]. In contrast, on $\mathrm{Cu}(111)$ the vertical distance of the perylene core from the $\mathrm{Cu}(111)$ surface is only $2.66 \AA$ [35]. This again points to a difference in the bonding character on the two surfaces.

Several studies have probed the influence of the adsorption on metal-supported hBN layers on the electronic structure of large organic molecules, namely their frontier orbitals, by PES [36] or STS [37,38]. However, to the best of our knowledge, there have been no studies on the FL of monolayers of molecules on metal-supported hBN layers, yet. Kerfoot et al. [22] studied the FL of PTCDA and perylene-3,4,9,10-tetracarboxylic-3,4,9,10diimide (PTCDI) on an exfoliated hBN monolayer that was transferred onto $\mathrm{SiO}_{2}$. Forker et al. [23] investigated the optical absorption properties of PTCDA on $\mathrm{hBN} / \mathrm{Rh}(111)$ and $\mathrm{hBN} /$ $\operatorname{Pt}(111)$.

Here, we report a direct comparison of FL spectra of PTCDA/ $\mathrm{hBN} / \mathrm{Cu}(111)$ and PTCDA/Cu(111), which allows for a relative determination of the efficiency of the $\mathrm{hBN}$ layer to decouple the excited states of PTCDA from $\mathrm{Cu}(111)$. For PTCDA on $\mathrm{Ag}(111)$ and $\mathrm{Au}(111)$ [39], it has been shown that FL can only be observed from the second and third molecular layer onward. The excitation of the first layers is completely quenched by the metal substrates as described above. In UPS experiments, a partial filling of the LUMO of PTCDA was found on $\operatorname{Ag}(111)$, but not on $\operatorname{Au}(111)$ [33]. Thus, the quenching on $\operatorname{Ag}(111)$ is directly understood by the static charge transfer seen in UPS. The quenching on $\mathrm{Au}(111)$, not as evident from UPS, demonstrates the sensitivity of FL spectroscopy to an overlap of wave functions of excited states. Accordingly, the same behavior as on $\mathrm{Ag}(111)$ can be expected on $\mathrm{Cu}(111)$, where a filling of the LUMO has been found, too [33]. In addition, for multilayer PTCDA films we can compare the first, interfacial PTCDA layer with a hBN layer regarding their abilities to decouple the next PTCDA layer from the $\mathrm{Cu}(111)$ substrate.

In this contribution, we will discuss Raman modes and several different FL lines of PTCDA that were observed on both hBN/ $\mathrm{Cu}(111)$ and $\mathrm{Cu}(111)$. For an effective comparison, the structures and the growth modes of the PTCDA layers are relevant. Details of the structural investigation of the two surfaces, including low-energy electron diffraction (LEED) patterns, are given in Appendix A. Since the interpretation of the optical data requires this knowledge, we summarize some details ahead here. PTCDA forms ordered structures and follows a layer-by- 
layer growth for at least the first three layers on $\mathrm{Cu}(111)$ and the first two layers on $\mathrm{hBN} / \mathrm{Cu}(111)$. We are able to distinguish PTCDA/hBN/Cu(111) from PTCDA/Cu(111), as well as the monolayer and multilayers of PTCDA on $\mathrm{Cu}(111)$ and $\mathrm{hBN} /$ $\mathrm{Cu}(111)$ by differences in the respective LEED patterns.

\section{Experimental}

All experiments were carried out in an ultrahigh-vacuum chamber with a base pressure of $2.3 \times 10^{-10}$ mbar. The $\mathrm{Cu}(111)$ sample could be heated up to $1100 \mathrm{~K}$ via a tungsten filament and electron bombardment and cooled down to $20 \mathrm{~K}$ by using liquid helium.

The hBN layer was grown by dosing the precursor borazine $\left[(\mathrm{HBNH})_{3}\right]$ into the chamber while the sample was held at 1010 K. Borazine was purchased from Katchem spol. s r. o. Czech Republic (http://www.katchem.cz). It was kept in a glass tube connected to the chamber via a dosing valve at constantly $-5{ }^{\circ} \mathrm{C}$. Prior to the $\mathrm{hBN}$ preparation, it was cleaned by three cycles of freezing the liquid borazine using liquid $\mathrm{N}_{2}$ and pumping away the gas phase above the frozen borazine. The composition of the gas phase in the borazine source was monitored by a quadrupole mass spectrometer (QMS). It was considered suitable for hBN preparation when the signals for $\mathrm{H}_{2}$ (a product of the decomposition of borazine that is known to occur even when stored at low temperatures, $\mathrm{m} / \mathrm{z} 2$ ) and borazine $(\mathrm{m} / \mathrm{z} 80)$ showed a ratio of approximately $1: 1$.

The clean $\mathrm{Cu}(111)$ surface was prepared by consecutive steps of sputtering for $30 \mathrm{~min}$ with $\mathrm{Ar}^{+}$ions $(1000 \mathrm{eV}, 4 \mu \mathrm{A})$ and annealing at $1010 \mathrm{~K}$ for $30 \mathrm{~min}$. After the last sputtering cycle, the $\mathrm{Cu}(111)$ sample was heated to $1010 \mathrm{~K}$ and ca. $2000 \mathrm{~L}$ borazine were dosed $\left(1.5 \times 10^{-6} \mathrm{mbar}\right.$ via the background for $30 \mathrm{~min}$ ) onto the sample held at $1010 \mathrm{~K}$. After stopping the borazine dosing, the sample was cooled down with $1 \mathrm{~K} \cdot \mathrm{s}^{-1}$. The structural quality of the bare $\mathrm{Cu}(111)$ surface, the hBN layers, and the PTCDA layers was checked by LEED. We used a SPALEED instrument as described in [30]. An additional annealing step between the last sputter cycle and the borazine deposition was omitted here in order to prevent segregation of chemical impurities from the $\mathrm{Cu}$ bulk to the surface, which we otherwise occasionally observed as additional LEED spots. The criteria for a good hBN layer were a sharp continuous ring of intensity with a radius that is $2 \%$ larger than the distance of the firstorder $\mathrm{Cu}$ spots from the specular spot and the appearance of a clear star-like pattern of satellites around the specular spot caused by multiple electron scattering as reported in [30].

PTCDA was evaporated from a glass crucible. The molecular flux was also monitored by the QMS at $m / z 392$, which corresponds to the mass of the non-fractured PTCDA molecule. The flux was typically one monolayer per minute. During deposition, the sample was held at a constant temperature. PTCDA layers on $\mathrm{Cu}(111)$ were prepared by keeping the sample at a temperature of either 20 or $300 \mathrm{~K}$ during deposition. PTCDA layers on $\mathrm{hBN} / \mathrm{Cu}(111)$ were prepared by deposition at a sample temperature of $20 \mathrm{~K}$ and subsequent annealing. The sample was annealed in iterative steps of heating, holding at a constant temperature for $3 \mathrm{~min}$, and cooling down again to $20 \mathrm{~K}$ for a measurement. The annealing temperatures were in the range of 100-400 K and are specified below. In the following, one monolayer (ML) of PTCDA refers to a single layer of closepacked, flat-lying PTCDA molecules. After every experiment, the exact coverage was determined by a thermally programmed desorption (TPD) experiment with a margin of error of $\pm 0.05 \mathrm{ML}$. The calibration for PTCDA/hBN/Cu(111) was derived from the TPD data shown in [32]. Since the packing densities of PTCDA on $\mathrm{hBN} / \mathrm{Cu}(111)$ and on $\mathrm{Cu}(111)$ are within a few percent of each other (see Appendix A), this calibration is also valid for PTCDA/Cu(111) within the noted margin of error.

For the optical experiments, the sample was transferred into a glass tube, standing out from the end of the chamber. In the glass tube, the sample was illuminated by a focused laser beam at an incident angle of approximately $45^{\circ}$ with respect to the surface normal. The diameter of the laser spot on the sample was about $0.5 \mathrm{~mm}$. The fluorescence and Raman scattered light from the sample was collected and parallelized by an achromatic lens and focused by a second achromatic lens onto the entrance slit of the spectrometer. The spectrometer (Acton, Spectra Pro 2300i, $f=0.3 \mathrm{~m}$ ) was operated with three different gratings $(300,600$, and 1200 grooves per millimeter, yielding a resolution of 48,24 , and $12 \mathrm{~cm}^{-1}$, respectively). It was equipped with a nitrogen-cooled CCD camera, operated at $-110{ }^{\circ} \mathrm{C}$. In order to block external stray light from entering the spectrometer, the glass tube, the lenses and the entrance slit of the spectrometer were enclosed by a black box. If not specified otherwise, we used an optically pumped cw semiconductor laser (Coherent Sapphire LP UBB CDRH) with a wavelength of $458 \mathrm{~nm}$ (photon energy of $2.698 \mathrm{eV}$ or $21,816 \mathrm{~cm}^{-1}$ ) and $P=50 \mathrm{~mW}$. To block the laser light from entering the spectrometer, a long-pass filter (cut-off at $475 \mathrm{~nm}$ ) was positioned in front of the entrance slit of the spectrometer. All spectra were recorded at a sample temperature of $20 \mathrm{~K}$ with an exposure time of $2 \mathrm{~s}, 50$ accumulations, and a slit width of the spectrometer of $0.1 \mathrm{~mm}$.

\section{Results and Discussion 1 Overview of the spectra}

Figure 2 shows overview spectra of 1.55 ML PTCDA on $\mathrm{Cu}(111)$ (red spectrum) and 0.50 ML PTCDA on $\mathrm{hBN} / \mathrm{Cu}(111)$ 
(blue spectrum). PTCDA on $\mathrm{Cu}(111)$ was deposited at a sample temperature of $300 \mathrm{~K}$ and the LEED pattern confirmed the formation of a second layer (see Appendix A). PTCDA on $\mathrm{hBN} / \mathrm{Cu}(111)$ was deposited at a sample temperature of $20 \mathrm{~K}$ and subsequently annealed at $300 \mathrm{~K}$. The FL spectrum shown here is identical to the FL spectrum of PTCDA deposited at a sample temperature of $300 \mathrm{~K}$, which showed the LEED pattern in Figure 8b (see below in Appendix A), revealing an ordered structure. Hence, we conclude that the blue FL spectrum shown in Figure 2, which is, as it will become clear, relevant for the comparison with PTCDA/Cu(111), also stems from an ordered PTCDA layer on $\mathrm{hBN} / \mathrm{Cu}(111)$.

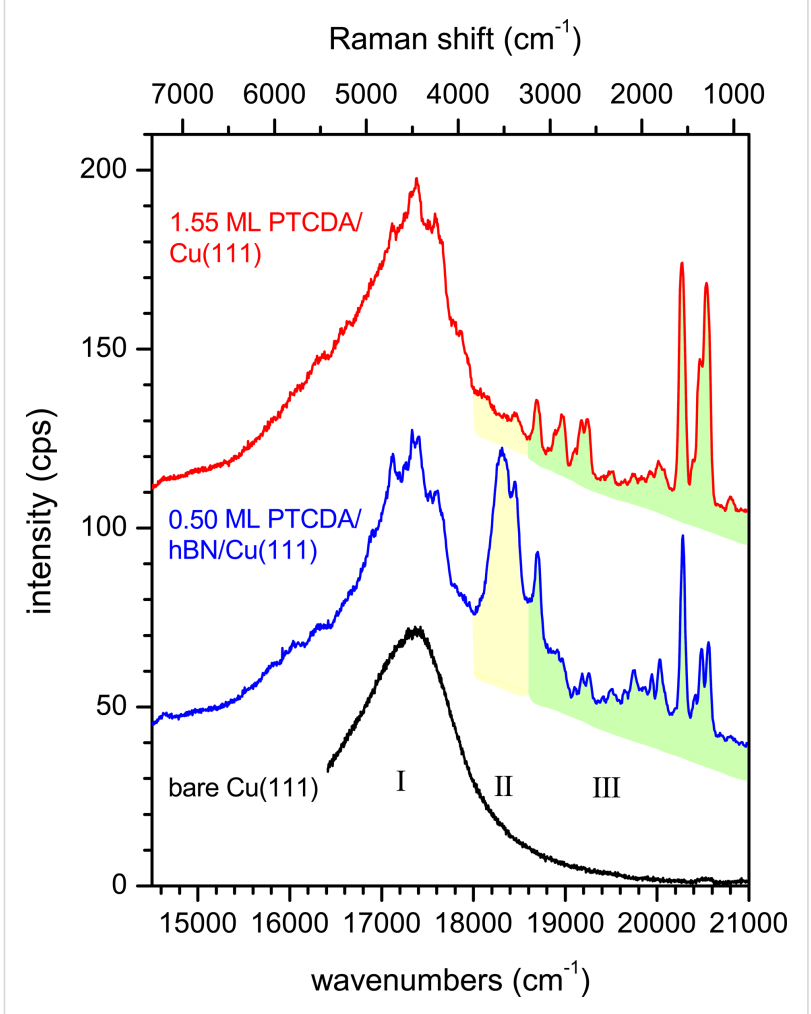

Figure 2: Overview spectra of 1.55 ML PTCDA on Cu(111) (red), of $0.50 \mathrm{ML}$ PTCDA on $\mathrm{hBN} / \mathrm{Cu}(111)$ (blue), and of the clean $\mathrm{Cu}(111)$ surface (black). For preparation details, see text. We distinguish three regions I, II, and III. The sharp lines marked in green are Raman modes (region III). The features marked in yellow are assigned to fluorescence (region II). The broadest feature on the low-energy side is due to defect luminescence of the Cu substrate (region I). All spectra were measured at $20 \mathrm{~K}$ using a grating with 300 grooves per millimeter. The spectra are shifted vertically for clarity.

For the discussion of the spectra, we consider three regions (I-III). At first glance, two of these regions appear qualitatively rather equal for both substrates: On the low-energy side below $18,000 \mathrm{~cm}^{-1}$ (region I) a broad luminescence can be observed and on the high-energy side above $18,600 \mathrm{~cm}^{-1}$ (region III), there is a set of sharp peaks. However, the two spectra differ significantly between 18,000 and $18,600 \mathrm{~cm}^{-1}$ (region II) due to broad FL peaks (highlighted in yellow) present for hBN/ $\mathrm{Cu}(111)$, but absent for PTCDA/Cu(111).

We tentatively assign the broad peak in region I to a radiative decay of interband transitions from the $\mathrm{Cu}$ substrate, as it can also be observed on the clean substrates $(\mathrm{Cu}(111)$ and $\mathrm{hBN} /$ $\mathrm{Cu}(111)$ ) before PTCDA deposition when all other features are absent (see Figure 2, black spectrum). An enhancement of radiative interband transitions has been reported for $\mathrm{Cu}$ nanoparticles [40]. We thus speculate that surface defects (protrusions) play a role here. This is in agreement with our observation that the intensity of this "defect luminescence" in region I depends on the exact position of the spot of the excitation light on the sample. It will not be in the focus of this work. The sharp peaks in region III are identified as Raman lines from PTCDA (highlighted in green). The Raman lines were additionally identified by using a dye laser with tunable wavelength [20] (497-507 nm). These peaks shift according to the wavelength of the laser (see Appendix B). Notably, some Raman peaks are superposed in the region of the defect luminescence of the $\mathrm{Cu}(111)$ surface (region I), too.

\section{Raman modes}

First, we will discuss the Raman lines. The peaks in the spectrum between 21,000 and $18,600 \mathrm{~cm}^{-1}$ in Figure 2 exhibit Raman shifts that correspond to the energies of the vibrational modes of PTCDA adsorbed on surfaces observed before $[41,42]$. The vibronic modes of PTCDA that can be observed in Raman spectroscopy are $A_{g}, B_{1 g}, B_{2 g}$, and $B_{3 g}$ modes, with the most prominent modes being $\mathrm{A}_{\mathrm{g}}$ modes between 1,250 and $1,650 \mathrm{~cm}^{-1}$ [41-43]. The spectral positions of most of these modes are about constant for PTCDA adsorbed on different substrates [41] or for different film thicknesses [42]. An interesting exception was observed for PTCDA on $\operatorname{Ag}(111)$ [42] for the breathing mode of the central carbon ring at ca. $1,300 \mathrm{~cm}^{-1}$. In the following, we will refer to it as ring breathing (RB) mode. On $\operatorname{Ag}(111)$, two different adsorption states of PTCDA were observed. Both states are bonded chemisorptively to the surface [44]. The RB mode of PTCDA deposited at a low temperature (LT) of $180 \mathrm{~K}$ exhibits a Raman shift of $1,310 \mathrm{~cm}^{-1}$. This is higher in energy by $13 \mathrm{~cm}^{-1}$ compared to the Raman shift $\left(1,297 \mathrm{~cm}^{-1}\right)$ for the RB mode of a layer at room temperature (RT) [42]. The special role of this RB mode will be discussed in further detail below.

\subsection{Surface-enhanced Raman scattering}

The fact that the Raman modes of a small quantity of molecules can be observed here at all is attributed to surface-enhanced Raman scattering (SERS) [45]. This effect is most commonly observed on rough surfaces of noble metals [45] or at metal nanostructures [46], and it is utilized in surface-enhanced 
Raman spectroscopy [47]. There are two explanations for it, namely, a chemical mechanism and an electromagnetic mechanism, which is thought to be the dominant contribution to the enhancement. The chemical mechanism is related to the specific chemical surface bonding of the investigated system. At its heart, a CT between the molecule and the substrate occurs due to the chemisorptive bonding, which leads to a change in the polarizability of the molecule and thus to an enhancement of the Raman signal. It is also possible that electronic excitations of the adsorbed molecule allow for a resonance Raman effect, which causes an additional enhancement [46]. According to the electromagnetic mechanism, on a rough surface, surface plasmon polaritons (SPPs) can also be excited by the incident light. The surface plasmons are located in the vicinity of surface defects, such as protrusions. The field enhancement at these defects leads to an enhancement of the Raman scattering [48]. Subsequently, the scattered light can be enhanced in the same manner. The electromagnetic mechanism may be responsible for an enhancement of the signal by a factor of $10^{5}-10^{6}$ [48]. The contribution of the chemical mechanism is generally much smaller, causing an enhancement by a factor of not more than $10^{2}-10^{3}[46]$.

Recently, hBN has gained interest as a SERS substrate [49]. In a comparative study on $2 \mathrm{DMs}$ on $\mathrm{SiO}_{2}$ it was shown that hBN had an enhancement effect on the Raman modes of adsorbed copper phthalocyanine molecules [50]. The effect was explained by the polar character of the $\mathrm{B}-\mathrm{N}$ bonds, which induced a dipole in the adsorbed molecule. The resulting interfacial dipole-dipole interactions are thought to have a similar effect on the polarizability of the adsorbed molecule as a CT.

Regarding the Raman enhancement effect of a noble metal surface, we mention a recent study by Stallberg et al. [39], which investigated optical spectra of PTCDA on $\operatorname{Ag}(111)$ and $\mathrm{Au}(111)$. They found Raman modes of PTCDA on the $\mathrm{Au}(111)$ surface, but not on the $\mathrm{Ag}(111)$ surface. This observation was discussed in view of the different energies of the SPPs of the two surfaces. Stallberg et al. used photon energies of $2.37 \mathrm{eV}$ on $\mathrm{Au}(111)$ and $2.43 \mathrm{eV}$ on $\mathrm{Ag}(111)$ and concluded that only the $\mathrm{SPP}$ of $\mathrm{Au}(111)$ located at $E_{\mathrm{SPP}}^{\mathrm{Au}}=2.5 \mathrm{eV}$ can resonantly interact with the incident light, leading to an enhancement of the Raman modes. The SPP on the $\operatorname{Ag}(111)$ surface has an energy of $E_{\mathrm{SPP}}^{\mathrm{Ag}}=3.7 \mathrm{eV}$. Hence, a resonance was considered less probable, yielding no enhancement of Raman modes. This model should evidently encompass that the coupling to the SPPs requires a rough surface or local protrusions on the surface due to defects that break the translational symmetry. For comparison, we note that the energy of the SPP of $\mathrm{Cu}(111)$, which is calculated from the condition $-\operatorname{Im}\left(\varepsilon^{-1}\right)=\varepsilon_{2} /\left(\varepsilon_{1}^{2}+\varepsilon_{2}^{2}\right)$ [51] using the dielectric functions given in [52], is obtained at a value of $E_{\mathrm{SPP}}^{\mathrm{Cu}}=2.3 \mathrm{eV}$. Our excitation energy was $2.698 \mathrm{eV}$. From our experiment we have indeed evidence that SERS is related to surface defects. This will be discussed in Section 2.3.

\subsection{Raman modes of PTCDA on hBN/Cu(111) and $\mathrm{Cu}(111)$}

Figure $3 \mathrm{a}$ and Figure $3 \mathrm{~b}$ show region III of the spectra of PTCDA on $\mathrm{hBN} / \mathrm{Cu}(111)$ and $\mathrm{Cu}(111)$ for Raman shifts of 1,240 to $1,660 \mathrm{~cm}^{-1}$ in detail. All Raman shifts are also summarized in Table 1. Some values are given as averages for different coverages as no trends as a function of the coverage were found (see caption). First, we discuss spectra of PTCDA on $\mathrm{hBN} / \mathrm{Cu}(111)$ and $\mathrm{Cu}(111)$, which were both stable under annealing at $300 \mathrm{~K}$ (cf. Figure 3a) and, hence, represent the final state. Kinetic effects do not play a role here. In Section 2.2.2, we focus on temperature-dependent effects (cf. Figure $3 b)$.

2.2.1 The final state $-\mathbf{3 0 0} \mathrm{K}$ spectra: Figure 3a shows Raman modes of PTCDA/hBN/Cu(111) after deposition at $20 \mathrm{~K}$ and subsequent annealing at $300 \mathrm{~K}$ (blue), and of PTCDA/Cu(111) after deposition at $300 \mathrm{~K}$ (red). The positions of the Raman modes of 1 ML PTCDA/Ag(111) measured by Schneider and Wagner [42,53], which was deposited at a sample temperature of $400 \mathrm{~K}$, are indicated by green vertical bars for comparison. It is apparent from Figure $3 \mathrm{a}$ that the modes of PTCDA/hBN/ $\mathrm{Cu}(111)$ are systematically shifted to smaller energies by about $7 \mathrm{~cm}^{-1}$ compared to PTCDA/Cu(111). In contrast, the modes of PTCDA/Ag(111) [53] agree well with the modes of PTCDA/ $\mathrm{Cu}(111)$ within $0.3 \%$. An exception is the RB mode. This mode will be discussed separately below. The shift of the other modes to higher vibrational energies seen in comparison of hBN/ $\mathrm{Cu}(111)$ and the metal surfaces $\mathrm{Cu}(111)$ and $\mathrm{Ag}(111)$ can be linked to the different bonding of PTCDA to the surfaces. In UPS experiments [33], a chemisorptive interaction of the metal surfaces with the PTCDA molecule was found, leading to a (partial) filling of the former $\mathrm{LUMO}$ on $\mathrm{Ag}(111)$ and $\mathrm{Cu}(111)$. On PTCDA/hBN/Cu(111), no such chemisorptive bonding was observed in UPS [32]. We suppose that the energy of the vibrational modes recorded here are influenced by molecule-substrate interactions. The chemisorptive bond to the metal surface makes the intermolecular bonds harder, which causes the respective vibrational modes to increase in energy. For Raman shifts below $1,500 \mathrm{~cm}^{-1}$, this interpretation is also supported by the closer agreement of the Raman modes on $\mathrm{hBN} / \mathrm{Cu}(111)$ with those measured for PTCDA single crystals [55] (see Table 1). However, for modes above $1,570 \mathrm{~cm}^{-1}$, the situation is reversed and not yet understood.

As mentioned before, the significance of the RB mode has been reported for PTCDA/Ag(111) [53]. The energy of this mode in- 

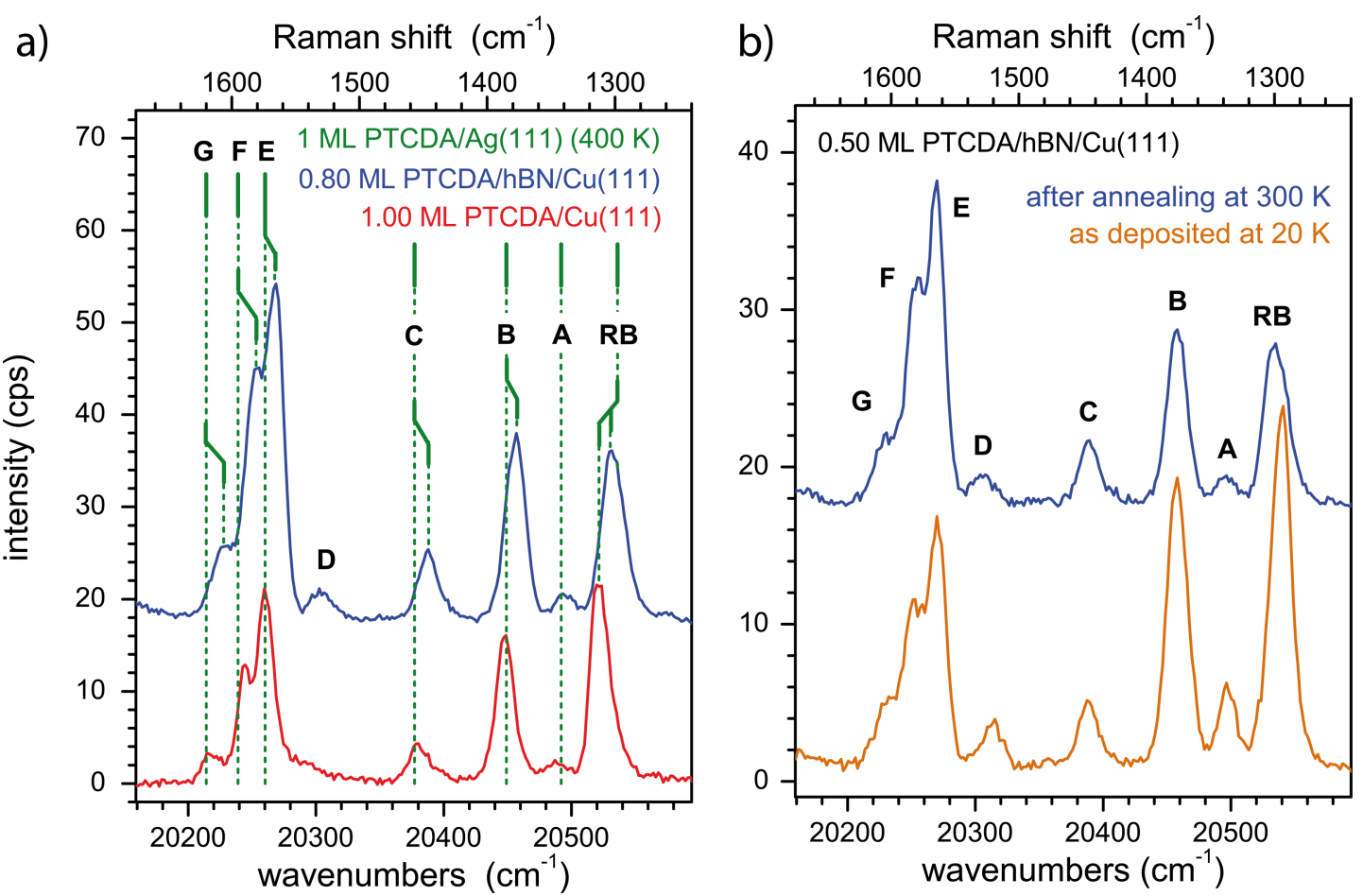

Figure 3: Zoom-in on the high-energy region (III) of the spectra. (a) $0.80 \mathrm{ML}$ PTCDA/hBN/Cu(111) after deposition at $20 \mathrm{~K}$ and subsequent annealing at $300 \mathrm{~K}$ (blue) and $1.00 \mathrm{ML}$ PTCDA/Cu(111) after deposition at $300 \mathrm{~K}$ (red). The positions of the Raman modes of $1 \mathrm{ML}$ PTCDA/Ag(111) [53] are indicated in green. (b) $0.50 \mathrm{ML} \mathrm{PTCDA} / \mathrm{hBN} / \mathrm{Cu}(111)$ after deposition at $20 \mathrm{~K}$ (orange) and after subsequent annealing at $300 \mathrm{~K}$ (blue). All spectra were measured at $20 \mathrm{~K}$ using a grating with 1200 grooves per millimeter. Labels of the peaks refer to Table 1 , the peaks $\mathrm{H}-\mathrm{K}$ are not shown here.

\begin{tabular}{|c|c|c|c|c|c|c|c|}
\hline mode & $\mathrm{hBN} / \mathrm{Cu}(111)$ & $\mathrm{hBN} / \mathrm{Cu}(111)$ & $\mathrm{Cu}(111)$ & $\mathrm{Cu}(111)$ & $\mathrm{Ag}(111)[53]$ & $\mathrm{KCl}[54]$ & PTCDA single crystal [55] ${ }^{a}$ \\
\hline$T_{\mathrm{S}}$ & $20 \mathrm{~K}$ & $300 \mathrm{~K}$ & $20 \mathrm{~K}$ & $300 \mathrm{~K}$ & $400 \mathrm{~K}$ & $<20 \mathrm{~K}$ & \\
\hline $\mathrm{RB}_{\text {mono }}$ & $1296.7 \pm 1.6$ & $1301.2 \pm 1.7$ & 1304.0 & 1312.9 & 1298 & 1288 & - \\
\hline $\mathrm{RB}_{\text {multi }}$ & $1301.8 \pm 3.8$ & $1309.8 \pm 3.1$ & 1300.0 & 1309.5 & 1309 & - & 1302.3 \\
\hline A & $1339.4 \pm 1.5$ & $1339.2 \pm 1.2$ & n.o. & 1346.5 & 1342 & 1332 & 1335.0 \\
\hline B & $1378.0 \pm 1.0$ & $1377.5 \pm 1.5$ & 1383.8 & 1386.0 & 1385 & 1368 & $1375.4 / 1383.6$ \\
\hline C & $1447.8 \pm 1.5$ & $1447.4 \pm 1.7$ & n.o. & 1454.6 & 1457 & 1446 & 1451.0 \\
\hline D & $1522.4 \pm 2.1$ & $1529.4 \pm 0.7$ & n.o. & n.o. & - & 1523 & - \\
\hline$E$ & $1566.6 \pm 1.9$ & $1567.9 \pm 2.5$ & 1571.4 & 1574.0 & 1574 & 1564 & 1570.6 \\
\hline $\mathrm{F}$ & $1582.7 \pm 3.7$ & $1582.3 \pm 2.0$ & n.o. & 1589.6 & 1595 & 1584 & 1589.1 \\
\hline G & $1606.7 \pm 1.7$ & $1607.3 \pm 2.0$ & n.o. & 1616.2 & 1620 & - & 1615.0 \\
\hline $\mathrm{H}$ & $1674.3 \pm 0.7$ & $1673.3 \pm 2.4$ & n.o. & n.o. & - & - & - \\
\hline I & $1757.8 \pm 1.8$ & $1758.0 \pm 1.7$ & n.o. & 1764.3 & - & - & - \\
\hline J & $1795.0 \pm 1.3$ & $1796.2 \pm 2.2$ & n.o. & n.o. & - & - & 1783.0 \\
\hline $\mathrm{K}$ & $1820.4 \pm 1.2$ & $1825.9 \pm 1.3$ & n.o. & 1833.7 & - & - & - \\
\hline
\end{tabular}

aOnly the upper Davydov components are listed. 
creases for PTCDA on different substrates, going from $\mathrm{KCl}$ films on $\operatorname{Ag}(100)\left(1,288 \mathrm{~cm}^{-1}\right.$, derived from FL spectra) [54], to $\operatorname{Ag}(111)\left(1,298 \mathrm{~cm}^{-1}\right)[53], \mathrm{hBN} / \mathrm{Cu}(111)\left(1,301 \mathrm{~cm}^{-1}\right.$, derived from FL spectra), and $\mathrm{Cu}(111)\left(1,313 \mathrm{~cm}^{-1}\right.$, derived from FL spectra), contrary to the other modes. This trend neither conforms with the strength of the (chemisorptive) bond to the substrate surface [33], nor with the bonding distance, or the amount of molecular distortion [32,35,56,57]. However, in some way it reflects the change in the distortion motif of the PTCDA molecule. On $\mathrm{KCl} / \mathrm{Ag}(100)$, the PTCDA molecule is bend like an arch with all of the oxygen atoms pulled towards the surface [57]. On $\operatorname{Ag}(111)$, the molecule is saddle-shaped with only the carboxylic oxygen atoms pulled downwards out of the molecular plane towards the surface [56]. On $\mathrm{Cu}(111)$, the opposite is found. The oxygen atoms are pushed away from the surface, upwards out of the molecular plane, leading to a boat shape [35]. On $\mathrm{hBN} / \mathrm{Cu}(111)$, the PTCDA molecule is nearly undistorted and hence planar [32]. It was surmised that the PTCDA molecule is bonded to the $\operatorname{Ag}(111)$ surface (and the $\mathrm{KCl}$ surface [57]) via the carboxylic oxygen atoms, while on $\mathrm{Cu}(111)$, the bonding proceeds primarily via the perylene core [58]. We hence suggest that the bonding via the perylene core on $\mathrm{Cu}(111)$ leads to a reduced flexibility of the intramolecular bonds of the core (including the central carbon ring), which causes the higher Raman shift of the RB mode. The flat, saddle-, and arch-like shapes of the molecule on $\mathrm{hBN} / \mathrm{Cu}(111)$, $\mathrm{Ag}(111)$, and $\mathrm{KCl} / \mathrm{Ag}(100)$ lead to smaller Raman shifts of the $\mathrm{RB}$ mode of $12 \mathrm{~cm}^{-1}, 15 \mathrm{~cm}^{-1}$, and $25 \mathrm{~cm}^{-1}$, respectively. The special sensitivity of the RB mode to the interfacial bonding was also seen in high-resolution electron loss spectra [59].

We also compare with PTCDA multilayers. The Raman shifts of the multilayers was found at similar energies on all three substrates, that is, at $1,309.8 \mathrm{~cm}^{-1}$ for multilayers of PTCDA/hBN/ $\mathrm{Cu}(111)$, at $1,309.5 \mathrm{~cm}^{-1}$ for $2.55 \mathrm{ML}$ PTCDA/Cu(111), and at $1,309 \mathrm{~cm}^{-1}$ for $60 \mathrm{ML}$ PTCDA/Ag(111) [53]. This is in agreement with an identical interaction between the PTCDA multilayers as it is expected. The energy of the RB mode for the multilayers is between that of the monolayer on $\mathrm{hBN} / \mathrm{Cu}(111)$ $\left(1,301.2 \mathrm{~cm}^{-1}\right)$ and the monolayer on $\mathrm{Cu}(111)\left(1,312.9 \mathrm{~cm}^{-1}\right)$. Actually, it is rather close to the value seen on $\mathrm{Cu}(111)$ within $3 \mathrm{~cm}^{-1}$ (see Table 1). This may indicate that intermolecular interactions between adjacent layers in a multilayer also have a significant impact on the vibrational properties and cannot be neglected. We note that these values are slightly larger than those measured for PTCDA single crystals [55] or thick films [43].

2.2.2 Temperature induced changes in the Raman spectra: The unique behavior of the RB mode can also be seen in its dependency on the preparation temperature both for PTCDA/
$\mathrm{hBN} / \mathrm{Cu}(111)$ and PTCDA/Cu(111). We compare spectra recorded directly after deposition at $20 \mathrm{~K}$ with those after annealing at $300 \mathrm{~K}$ (on $\mathrm{hBN} / \mathrm{Cu}(111)$ ) (Figure 3b) or with those after deposition at $300 \mathrm{~K}$ (on $\mathrm{Cu}(111)$ ) (spectra not shown). For PTCDA deposited onto $\mathrm{Cu}(111)$ at $20 \mathrm{~K}$, no Raman peaks could be observed at all in the sub-monolayer regime, and even for multilayers, the intensities of the Raman modes did not exceed two counts per second. (These are the modes given in Table 1.) At higher temperatures, the RB mode shifts to higher energies by 4 and $9 \mathrm{~cm}^{-1}$ for PTCDA on hBN/Cu(111) and $\mathrm{Cu}(111)$, respectively; the other modes remain unchanged $( \pm 0.4 \%$, cf. Table 1).

This reflects the situation of PTCDA/Ag(111) [42] where the only significant temperature-dependent shift (by $10 \mathrm{~cm}^{-1}$ ) was also observed for the RB mode. In that study, the shift of the $\mathrm{RB}$ mode was originally attributed to be of chemical origin due to different bonding to the $\mathrm{Ag}(111)$ substrate present only in the monolayer but not for the multilayers. However, this interpretation appears less possible for the monolayers considered here. Hence, we recall a later study [56] that revealed the existence of a chemisorbed LT state of the monolayer in which the PTCDA molecule is positioned at a similar distance $( \pm 5 \%$, regarding the perylene core) from the surface as the RT state but is significantly more distorted than the RT state (maximum height difference of atoms of $0.31 \AA$ compared to $0.20 \AA$ ) [56]. In addition, the LT state is present as a disordered phase, while the RT state forms highly ordered domains [44]. This is accompanied by a change in the valence band structure as seen, for example, in UPS [44]. Thus, instead of a multilayer/monolayer effect, the above described differences in the Raman shifts may also be caused by the intermolecular interactions and a modification in the interfacial bonding in the ordered domains of the RT state, which form upon annealing at room temperature. We propose that, in particular, the effect of intermolecular interactions is also relevant for the monolayer of PTCDA on $\mathrm{hBN} / \mathrm{Cu}(111)$, whereas on $\mathrm{Cu}(111)$ concomitant changes in the interfacial bonding may play a role, too. The increased temperature induces the formation of ordered domains (observed in LEED). The intermolecular interactions in the domains then cause a change in the structure and charge distribution within the molecule. This in turn increases the vibrational energies, in particular of the RB mode located on the perylene core.

\subsection{The role of surface defects for SERS}

For both PTCDA/hBN/Cu(111) and PTCDA/Cu(111), the overall intensities of the Raman modes are of the same order, which implies that both surfaces cause a similar degree of Raman enhancement. However, we refrain from making a statement about the SERS effectiveness of $\mathrm{hBN}$ on $\mathrm{Cu}(111)$ for the following reason: In our experiments, the Raman intensities on 
either $\mathrm{hBN} / \mathrm{Cu}(111)$ or $\mathrm{Cu}(111)$ were both found to be highly dependent on the exact position of the incident laser beam on the sample (varying by a factor of up to ca. 7). This indicates that the specific local surface quality, for example, the surface roughness at the spot where the Raman scattering occurred, influences the intensity of the Raman modes. Hence, we cannot draw quantitative conclusions here. Nevertheless, we gain some insight into this aspect from an experiment we conducted at low temperatures, which we describe in the following.

As mentioned in the previous section, in the sub-monolayer regime of PTCDA/Cu(111) deposited at $20 \mathrm{~K}$ no Raman peaks could be observed at all, and even for multilayers their intensities were very small (spectra not shown). For PTCDA on hBN/ $\mathrm{Cu}(111)$ the situation is drastically different. Figure $3 \mathrm{~b}$ shows the Raman modes of PTCDA (0.50 ML) after deposition at $20 \mathrm{~K}$ (orange) and after subsequent annealing at $300 \mathrm{~K}$ (blue). For both preparations, the Raman modes are clearly present and of a similar intensity.

We discuss two possible explanations for these different kinds of behavior after deposition at $20 \mathrm{~K}$ : (i) The dipole-dipole interaction between hBN and PTCDA enhances the Raman signal. In this process, the underlying copper would not be involved [50]. (ii) The Raman modes of PTCDA are strongly enhanced at specific adsorption sites, which we refer to as "hot spots". On $\mathrm{Cu}(111)$, the molecules can reach these hot spots only by temperature-induced diffusion. Whereas, after deposition at $20 \mathrm{~K}$, the molecules stay statistically distributed on the surface, and only a small fraction is located at these sites where the SERS effect occurs. On $\mathrm{hBN} / \mathrm{Cu}(111)$, the significantly smaller interaction between PTCDA and the hBN surface compared to $\mathrm{Cu}(111)$ and a consequently smaller corrugation of the bonding potential lead to a much higher mobility, which allows for a diffusion to the hot spots even at low temperatures of $20 \mathrm{~K}$.

We propose the second interpretation to be relevant. Our arguments are the following: Firstly, we have shown in a previous work [32] that the interaction between PTCDA and hBN/ $\mathrm{Cu}(111)$ is of a physisorptive nature, which makes a strong SERS effect due to dipole-dipole interactions at the interface [50] unlikely. Secondly, the strong dependence of the Raman intensity on the sample position (see above) conforms with the interpretation of a SERS effect related to local hot spots. However, the details of the related adsorption sites remains unclear

Since the SERS effect is primarily observed on rough/nanostructured rather than on flat metal surfaces [45], the SERS effect is expected to be larger for molecules located in proximity to surface defects. Thus, at surface positions with a higher defect density, the Raman intensities are expected to be higher.
The relevance of the defect density in the present case is corroborated by our observation that at positions where the Raman intensity was high, the defect luminescence of the substrate (see region I in Figure 2) was also higher. In a theoretical study, García-Vidal and Pendry investigated the enhancement due to the SERS effect as a function of the roughness on a surface [48]. They found that on an inhomogeneously rough surface, the Raman spectrum is dominated by the enhanced signals from molecules located at features that exhibit a maximum in roughness. We can support this interpretation by results from a previous experiment. We observed by light microscopy that $\mathrm{Ag}(100)$ samples that had been prepared in a similar manner as the $\mathrm{Cu}(111)$ surface used here and that yielded a high-quality LEED pattern indicating the presence of long-range ordered and large, defect-free terraces, actually show variations in their surface morphology on a micrometer scale [60]. We propose that the defect-rich regions exhibit a large step density due to impurities and/or grain boundaries. Consequently, we assume that the hot spots are related to an inhomogeneous mesoscopic roughness of the $\mathrm{Cu}(111)$ surface, which is remnant after sputtering and annealing.

In conclusion, the Raman lines of PTCDA/hBN/Cu(111) and PTCDA/Cu(111) observed between 18,600 and $21,000 \mathrm{~cm}^{-1}$ are in accordance with the characteristic fingerprint of the molecule. They can be used as a monitor for its presence on the surfaces. Coverages cannot be determined quantitatively from the intensities of the Raman modes, since these depend on the sample positions, which we assign to a correlation with the local roughness due to structural defects of the surface. Note that we systematically did not observe the characteristic Raman line of $\mathrm{hBN}$ at approximately $1,370 \mathrm{~cm}^{-1}$ that was observed for $\mathrm{hBN}$ on $\mathrm{SiO}_{2} / \mathrm{Si}$ [61], on $\mathrm{Cu}$ foils [62], and on other metal foils $[63,64]$ for our samples of $\mathrm{hBN} / \mathrm{Cu}(111)$. This is an obvious discrepancy, which we cannot explain based on our current data. It may, however, be related to the specific interface between $\mathrm{hBN}$ and the single crystalline $\mathrm{Cu}$ substrate.

\section{Fluorescence}

\subsection{PTCDA/hBN/Cu(111)}

We turn to the FL, which can be observed in region II of Figure 2, between 18,000 and $18,600 \mathrm{~cm}^{-1}$. We note ahead that we did not observe vibronic bands related to the FL peaks as it is the case, for example, for PTCDA on $\mathrm{KCl}$ [65].

First, we consider which experimental conditions led to the observation of FL peaks. Figure 4a shows a zoom-in on region II of the spectrum of $0.60 \mathrm{ML}$ PTCDA on $\mathrm{hBN} / \mathrm{Cu}(111)$ as deposited at a sample temperature of $20 \mathrm{~K}$, and after a series of subsequent annealing steps at temperatures between 100 and $400 \mathrm{~K}$. The peak on the high-energy side of the spectrum 
(marked in green and labeled " $\mathrm{R}$ ") is a Raman mode. This mode has also been observed for PTCDA/Ag(111) [53]. Furthermore, its change in intensity as a function of the annealing temperature agreed with that of other Raman modes. The behavior of the two other peaks depicted in Figure 4a (marked in yellow and pink) was quantitatively different. We assign these peaks to the FL of PTCDA molecules and will refer to them as $\mathrm{FL}_{\mathrm{A}}$ (at ca. $18,450 \mathrm{~cm}^{-1}$ ) and $\mathrm{FL}_{\mathrm{B}}$ (at ca. $18,300 \mathrm{~cm}^{-1}$ ). Both peaks appear after annealing at temperatures above ca. $200 \mathrm{~K}\left(\mathrm{FL}_{\mathrm{A}}\right)$ and ca. $280 \mathrm{~K}\left(\mathrm{FL}_{\mathrm{B}}\right)$.

We take this finding as an indication that the FL of PTCDA on $\mathrm{hBN} / \mathrm{Cu}(111)$ depends on the structural order of the molecules on the surface that is established by annealing. We explain the existence of two FL peaks by the presence of two structurally different "phases" of PTCDA. Since all optical measurements were carried out at a sample temperature of $20 \mathrm{~K}$, the temperature-induced structural ordering upon annealing that led to the FL peaks is irreversible. The broad FL peaks are superimposed with several sharp Raman lines, which lead to modulations of the peaks. There are two important differences between the $\mathrm{FL}_{\mathrm{A}}$ and $\mathrm{FL}_{\mathrm{B}}$ peaks: $\mathrm{FL}_{\mathrm{B}}$ is significantly broader than $\mathrm{FL}_{\mathrm{A}}$ (by a factor of ca. three, which we will discuss in detail in the Section Final Discussion). In addition, the intensity of $\mathrm{FL}_{\mathrm{A}}$ saturates, while that of $\mathrm{FL}_{\mathrm{B}}$ does not. Notably, the intensity of $\mathrm{FL}_{\mathrm{A}}$ saturates upon annealing at $280 \mathrm{~K}$, which is the temperature that is required for $\mathrm{FL}_{\mathrm{B}}$ to be observed at all. This behavior was found for layers within a range of sub-monolayer coverages (between 0.10 and $0.80 \mathrm{ML}$ ).

For further insight, we refer to a temperature-dependent series of spectra at a higher coverage of 1.55 ML PTCDA on hBN/ $\mathrm{Cu}(111)$, shown in Figure 4b. We observe the same Raman line as for the $0.60 \mathrm{ML}$ spectrum, as well as $\mathrm{FL}_{\mathrm{A}}$ and $\mathrm{FL}_{\mathrm{B}}$. However, there are a few important differences compared to the spectra in Figure 4a: (i) $\mathrm{FL}_{\mathrm{A}}$ can be observed in the spectrum immediately after deposition at $20 \mathrm{~K}$; (ii) $\mathrm{FL}_{\mathrm{B}}$ appears in the spectrum already after annealing at $200 \mathrm{~K}$ (not at $280 \mathrm{~K}$ ); and (iii) additional FL intensity at lower energies (ca. 18,150 $\mathrm{cm}^{-1}$ ) appears, which we assign to a third peak $\mathrm{FL}_{\mathrm{C}}$. The appearance of $\mathrm{FL}_{\mathrm{A}}$ at low temperatures leads us to the conclusion that the attributed "phase A" of PTCDA/hBN/Cu(111) forms directly at higher coverages, while at lower coverages its formation requires annealing. Hence, we assign $\mathrm{FL}_{\mathrm{A}}$ to PTCDA molecules at surface defects. In a sub-monolayer, the molecules can reach these defects via diffusion, which has to be temperatureinduced. For a higher coverage, the sites at defects are already populated during the deposition, even at low temperatures. A saturation of the sites at defects leads to the observed intensity saturation of the corresponding $\mathrm{FL}_{\mathrm{A}}$ peak. We note in this context that the surface defects connected to $\mathrm{FL}_{\mathrm{A}}$ are not identical to the hot spots mentioned above, which support the SERS
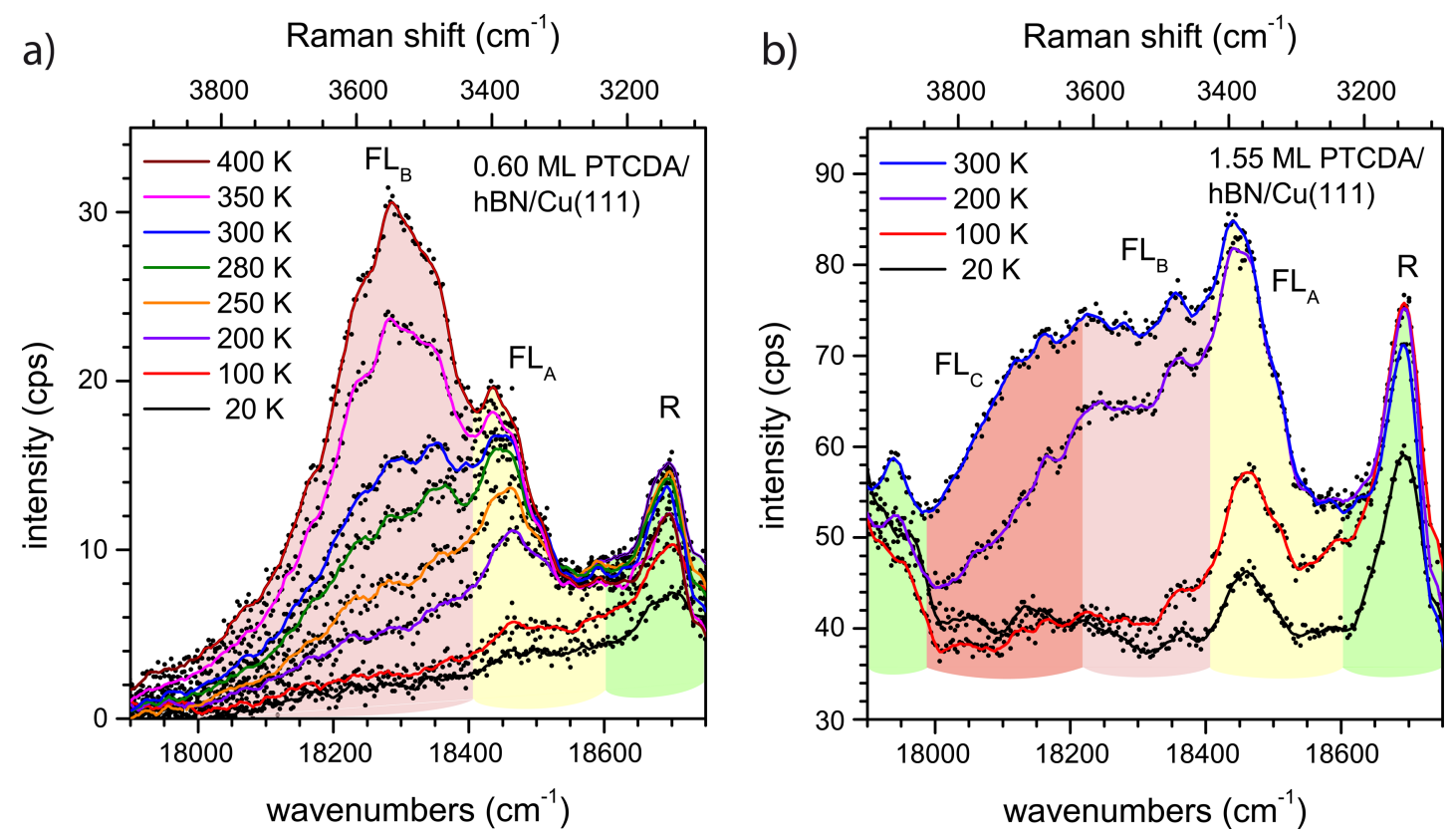

Figure 4: Fluorescence spectra of (a) $0.60 \mathrm{ML}$ PTCDA and (b) $1.55 \mathrm{ML}$ PTCDA on hBN/Cu(111) as deposited at $20 \mathrm{~K}$ (black) and subsequently annealed at different temperatures. Raman modes are highlighted in green, $F L$ peaks $F L_{A}$, $F L_{B}$, and $F L_{C}$ are highlighted in yellow, pink, and red, respectively. The spectrum of the clean surface was subtracted as a background. Spectra are smoothed (lines, original data as dots). All spectra were measured at $20 \mathrm{~K}$ using a grating with 600 grooves per millimeter. 
effect, as we will discuss below. After the saturation of the defects, the temperature-induced formation of a second "phase B" occurs leading to the peak $\mathrm{FL}_{\mathrm{B}}$.

We also observed the $\mathrm{FL}_{\mathrm{B}}$ peak on $\mathrm{hBN} / \mathrm{Cu}(111)$ after deposition at $300 \mathrm{~K}$ for coverages of 1-3 ML. For all these experiments, we observed the LEED pattern that is characteristic of ordered domains of PTCDA/hBN/Cu(111) (see Appendix A). Thus, we conclude that $\mathrm{FL}_{\mathrm{B}}$ stems from these ordered PTCDA domains in the first layer on hBN. The formation of ordered domains is not possible at a sample temperature of $20 \mathrm{~K}$ during deposition. It requires a certain threshold temperature (ca. $280 \mathrm{~K}$ ). This explains why $\mathrm{FL}_{\mathrm{B}}$ can only be observed after annealing or deposition at or above this temperature.

The energetic difference between $\mathrm{FL}_{\mathrm{A}}$ and $\mathrm{FL}_{\mathrm{B}}$ amounts to ca. $150 \mathrm{~cm}^{-1}$. We compare this to the energetic shift reported by Forker et al. who investigated PTCDA on hBN/Rh(111) [23]. They found that PTCDA molecules on hBN/Rh(111) are trapped in the pores of the $\mathrm{hBN}$ superstructure, which leads to isolated monomers on the surface. Annealing leads to the formation of ordered domains and a redshift of the spectrum by $223 \mathrm{~cm}^{-1}$ (27.6 meV) [23]. The molecules trapped in pores on $\mathrm{hBN} / \mathrm{Rh}(111)$ can be compared with isolated molecules located at defects on $\mathrm{hBN} / \mathrm{Cu}(111)$. However, please note that we do not consider trapped PTCDA molecules but isolated molecules to be the origin of $\mathrm{FL}_{\mathrm{A}}$. This will be discussed in further detail in Section 3.3 in relation with further information from FL experiments. An energetic shift of the same order has also been observed for the FL of isolated molecules and that of ordered domains of PTCDA on $\mathrm{NaCl}$ [10]. Here, two different structures of ordered domains of PTCDA were observed (a herringbone and a quadratic structure) the $\mathrm{S}_{0} / \mathrm{S}_{1}$ transitions of which are redshifted relative to the isolated molecules by $560 \mathrm{~cm}^{-1}$ and $300 \mathrm{~cm}^{-1}$, respectively.

For a comparison of $\mathrm{FL}_{\mathrm{B}}$ of PTCDA domains on $\mathrm{hBN} / \mathrm{Cu}(111)$ at $18,300 \mathrm{~cm}^{-1}$, we refer to optical data taken for ordered monolayers of PTCDA on a monolayer of hBN grown on other substrates. The respective values are given in Table 2. Note that only $\mathrm{PTCDA} / \mathrm{hBN} / \mathrm{SiO}_{2}$ was investigated by FL spectroscopy while for PTCDA/hBN/Pt(111) and PTCDA/hBN/Rh(111) absorption spectra were measured. We cannot explain the differences of the $S_{0} / S_{1}$ transition energies, yet. However, we observe a trend of higher transition energies from $\mathrm{hBN} / \mathrm{SiO}_{2}$ to $\mathrm{hBN} / \mathrm{Cu}(111)$ to $\mathrm{hBN} / \mathrm{Pt}(111)$ to $\mathrm{hBN} / \mathrm{Rh}(111)$. This is the direction of increasing interactions between the hBN layer and the supporting metal substrate, as indicated by the increasing amplitude of the buckling of the hBN layers $[30,66]$. We note that Forker et al. [23] could exclude a dominant role of the dielectric properties of the metal substrates for the transition energies.

The peak $\mathrm{FL}_{\mathrm{C}}$ (cf. Figure $4 \mathrm{~b}$ ) shows the same behavior as $\mathrm{FL}_{\mathrm{B}}$ at a coverage of $1.55 \mathrm{ML}$. It is as broad as $\mathrm{FL}_{\mathrm{B}}$ and appears after annealing at $200 \mathrm{~K}$. However, it is not present at a coverage of 0.60 ML. Thus, it does not stem from PTCDA directly adsorbed on hBN, but from ordered PTCDA domains in a second layer. However, this is different from the FL emission (Y), which is present in bulk-like PTCDA layers and was reported, for example, in [67]. Indeed, we observed the broad Y line and its vibronic progression $\mathrm{Y}^{\prime}$ for higher coverages of 4-5 ML at 15,950 and $14,700 \mathrm{~cm}^{-1}$, respectively. This is in agreement with the line positions measured in [67] within $50 \mathrm{~cm}^{-1}$ and $300 \mathrm{~cm}^{-1}$, respectively.

In summary, for PTCDA/hBN/Cu(111) three FL peaks can be observed. $\mathrm{FL}_{\mathrm{A}}$ (ca. $18,450 \mathrm{~cm}^{-1}$ ) is present in the first layer and stems from molecules at surface defects. To enable the molecules to reach these defects, a temperature-induced diffusion is necessary at sub-monolayer coverage. $\mathrm{FL}_{\mathrm{B}}$ (ca. 18,300 $\mathrm{cm}^{-1}$ ) stems from ordered domains in the first PTCDA layer. It can only form under deposition at a sample temperature above ca. $280 \mathrm{~K}$, or after annealing at this temperature. $\mathrm{FL}_{\mathrm{C}}$ (ca. $18,150 \mathrm{~cm}^{-1}$ ) is assigned to the FL from ordered PTCDA domains in the second or higher layers.

\subsection{Comparison with PTCDA/Cu(111)}

Figure 5a shows the FL spectra of PTCDA/hBN/Cu(111), prepared by deposition at $20 \mathrm{~K}$ and subsequent annealing at $300 \mathrm{~K}$ and of PTCDA/Cu(111), prepared by deposition at $300 \mathrm{~K}$, both with varying coverages. We will list the important observations

Table 2: Spectral positions of the $\mathrm{S}_{0} / \mathrm{S}_{1}$ transition of PTCDA domains in the first monolayer on $\mathrm{hBN}$ on $\mathrm{SiO}$ [22], $\mathrm{Cu}(111), \mathrm{Pt}(111)$ [23], and $\mathrm{Rh}(111)$ [23]. PTCDA on $\mathrm{hBN} / \mathrm{SiO}_{2}$ and on $\mathrm{hBN} / \mathrm{Cu}(111)$ were investigated using FL spectroscopy. The results for $\mathrm{PTCDA}$ on $\mathrm{hBN} / \mathrm{Pt}(111)$ and $\mathrm{hBN} / \mathrm{Rh}(111)$ were gained in absorption experiments.

\begin{tabular}{llll}
$\mathrm{hBN} / \mathrm{SiO}_{2}[22]$ & $\mathrm{hBN} / \mathrm{Cu}(111)$ & $\mathrm{hBN} / \mathrm{Pt}(111)[23]$ & $\mathrm{hBN} / \mathrm{Rh}(111)[23]$ \\
\hline $2.234 \mathrm{eV}$ & $2.26 \mathrm{eV}$ & $2.31 \mathrm{eV}$ & $2.38 \mathrm{eV}$ \\
$18,060 \mathrm{~cm}^{-1}$ & $18,300 \mathrm{~cm}^{-1}$ & $18,700 \mathrm{~cm}^{-1}$ & $19,200 \mathrm{~cm}^{-1}$
\end{tabular}


for these spectra: (i) There is no fluorescence at all for a full monolayer of PTCDA on bare $\mathrm{Cu}(111)$ while on $\mathrm{hBN} / \mathrm{Cu}(111)$ FL can be observed for a coverage as low as 0.10 ML. (ii) In the second monolayer of PTCDA on bare $\mathrm{Cu}(111)$, an FL peak appears at $18,450 \mathrm{~cm}^{-1}$, which corresponds to $\mathrm{FL}_{\mathrm{A}}$. (iii) In the third PTCDA layer on $\mathrm{Cu}(111)$, there is an FL peak at
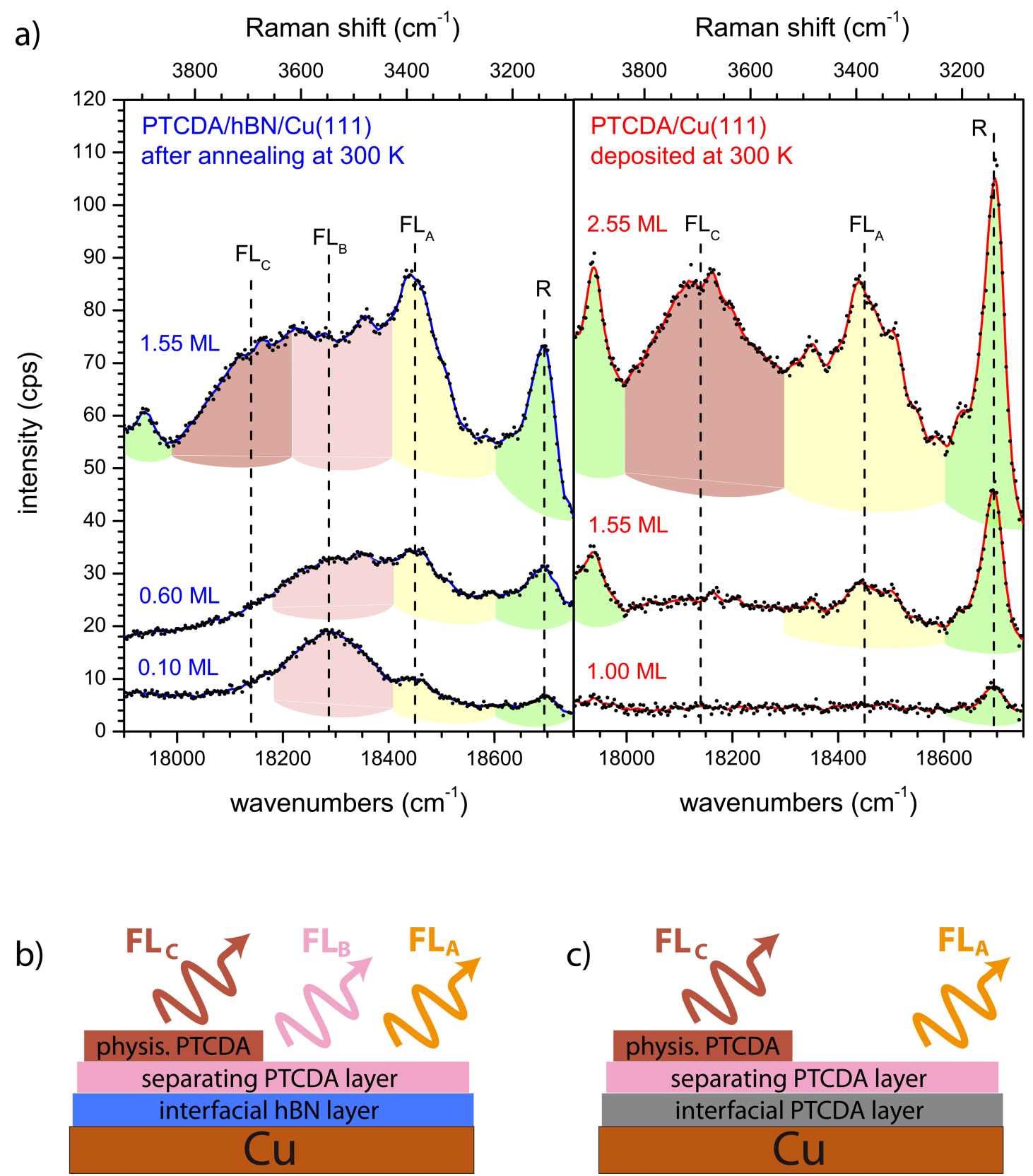

Figure 5: (a) Fluorescence spectra of PTCDA on hBN/Cu(111) (left, blue spectra, prepared by deposition at $20 \mathrm{~K}$ and subsequent annealing at $300 \mathrm{~K}$ ) and PTCDA/Cu(111) (right, red spectra, prepared by deposition at $300 \mathrm{~K}$ ). Raman intensities are highlighted in green; $F L_{\text {peaks }} \mathrm{FL}_{\mathrm{A}}$, $\mathrm{FL}_{\mathrm{B}}$, and $\mathrm{FL}_{\mathrm{C}}$ are highlighted in yellow, pink, and red, respectively. There is no FL from ordered domains in the first or second layers of PTCDA/Cu(111), but from the first layer of PTCDA/hBN/Cu(111). Spectra are smoothed (lines) and vertically shifted (original data as dots). Spectra of the clean substrates were subtracted as background. All spectra were measured at $20 \mathrm{~K}$ using a grating with 600 grooves per millimeter. (b) Schematic representations of $2 \mathrm{ML}$ $\mathrm{PTCDA} / \mathrm{hBN} / \mathrm{Cu}(111)$ and $(\mathrm{c}) 3 \mathrm{ML} \mathrm{PTCDA} / \mathrm{Cu}(111)$ and the $\mathrm{FL}$ signals observed from these layers. The FL from the first PTCDA layer on Cu is quenched (gray). The interfacial hBN layer (blue) and the interfacial chemisorbed PTCDA layer (gray) decouple the fluorescing molecules from the metal. Defect-mediated FL $\left(F L_{A}\right)$ is emitted from the separating PTCDA layer (pink) in both cases, FLC originates from the physisorbed PTCDA layer (red). Only for PTCDA/hBN/Cu(111) FL occurs from PTCDA domains in the separating layer (FL $\mathrm{B}_{\mathrm{B}}$ ). For details on the different PTCDA layers, see text. 
$18,150 \mathrm{~cm}^{-1}$, which is the position of $\mathrm{FL}_{\mathrm{C}}$ observed for 1.55 ML PTCDA/hBN/Cu(111). (iv) $\mathrm{FL}_{\mathrm{B}}$ is not observed at all for PTCDA/Cu(111). The absence of $\mathrm{FL}_{\mathrm{B}}$ on $\mathrm{Cu}(111)$ is understood as we have assigned it to PTCDA domains on hBN/ $\mathrm{Cu}(111)$. The other three observations have to be explained.

Ad (i): The absence of any FL of 1.00 ML PTCDA on $\mathrm{Cu}(111)$ confirms the complete quenching of the molecular excitation in the first layer on a metal surface as has previously been observed for the $\mathrm{Ag}(111)$ [5,39] and the $\mathrm{Au}(111)$ [39] surfaces. Ad (ii): For PTCDA/Cu(111), a sufficient decoupling to allow some FL is achieved for the second layer (1.55 ML). Thus, $\mathrm{FL}_{\mathrm{A}}$ can be observed, which we assigned to FL from molecules at defects on the $\mathrm{hBN} / \mathrm{Cu}(111)$ surface. It is remarkable that the PTCDA molecules have to interact with defects in order to fluoresce, and that the same kind of FL can be observed on both substrates. For PTCDA on $\mathrm{Cu}(111)$, as on $\mathrm{hBN} / \mathrm{Cu}(111)$, a temperature-induced process is necessary to observe $\mathrm{FL}_{\mathrm{A}}$ as PTCDA on $\mathrm{Cu}$ deposited at $20 \mathrm{~K}$ shows no FL at all (not shown). Only for PTCDA deposited at $300 \mathrm{~K}$ on $\mathrm{Cu}(111)$, we can observe the spectra shown in Figure 5. As stated in the previous section, we assume a temperature-induced diffusion process to surface defects where the PTCDA molecules are then able to fluoresce. The nature of these defects will be discussed in the next Section 3.3. Ad (iii): From our LEED experiments (see Appendix A) we know that PTCDA on $\mathrm{Cu}(111)$ grows layer-by-layer in the first three layers. Thus, we can assign the peak $\mathrm{FL}_{\mathrm{C}}$, which appears in the third layer, to FL from ordered PTCDA domains adsorbed on top of two completed PTCDA layers. As we will discuss now, this is in accordance with the observation of $\mathrm{FL}_{\mathrm{C}}$ from the second layer of PTCDA/hBN/ $\mathrm{Cu}(111)$. We summarize our observations with the schematic layer models that are given in Figure 5b,c. In both cases an interfacial layer that is adsorbed on the $\mathrm{Cu}(111)$ surface exists. This layer decouples PTCDA molecules in higher layers, which leads to FL from these layers. The interfacial layer is either a layer of hBN or a monolayer of chemisorbed PTCDA. The next layer in both cases is formed by ordered PTCDA domains that show $\mathrm{FL}_{\mathrm{A}}$, likely from molecules at defects. In the case of PTCDA on $\mathrm{hBN} / \mathrm{Cu}(111)$, an additional $\mathrm{FL}_{\mathrm{B}}$ from ordered domains is present. We call this first PTCDA layer a separating layer. In the TPD spectrum of PTCDA/hBN/Cu(111), the desorption peak of the separating layer can be distinguished from that of the multilayers (however, no separated peak for the second layer is observed) [32]. On $\mathrm{Cu}(111)$, the first (interfacial) layer does not desorb at all, and in the TPD spectrum, the second (separating) layer is also distinct from the multilayers [68]. The multilayers (both on $\mathrm{hBN} / \mathrm{Cu}(111)$ and $\mathrm{Cu}(111)$ ) are likely physisorbed and include the layers from the second and third layer onward. The ordered PTCDA domains in these layers are the origin of $\mathrm{FL}_{\mathrm{C}}$.
Our findings are in accord with the conclusions by Stallberg et al. who measured photoluminescence spectra of PTCDA on $\mathrm{Ag}(111)$ and $\mathrm{Au}(111)$ [39]. From coverages of $1.8 \mathrm{ML}$ on $\mathrm{Ag}(111)$ and $2.2 \mathrm{ML}$ on $\mathrm{Au}(111)$ onward they observed FL peaks at $17,000 \mathrm{~cm}^{-1}$ and $17,300 \mathrm{~cm}^{-1}$ (denoted by $\mathrm{M}$ in [39]). These were assigned to PTCDA layers separated from the metal substrate by the first interfacial PTCDA layer. This M line was distinct from that of the bulk-like FL emission from thick films, which was found at $15,000 \mathrm{~cm}^{-1}$ (Y line) and $13,700 \mathrm{~cm}^{-1}$ (E line). The $\mathrm{M}$ line was observed even for multilayers, co-existing with the bulk emission. Similarly, for PTCDA/ $\mathrm{Cu}(111)$, we observed the $\mathrm{Y}$ line and its vibronic progression $\mathrm{Y}^{\prime}$ of the multilayer FL for a coverage of $4.60 \mathrm{ML}$ at $16,100 \mathrm{~cm}^{-1}$ and $14,750 \mathrm{~cm}^{-1}$, respectively, while $\mathrm{FL}_{\mathrm{A}}$ and $\mathrm{FL}_{\mathrm{C}}$ were still present (not shown). The $\mathrm{Y}$ line was assigned to the $0-0$ transition of the PTCDA bulk phase [67]. Thus, the co-existence of the $\mathrm{Y}$ line and $\mathrm{FL}_{\mathrm{A}}$ and $\mathrm{FL}_{\mathrm{C}}$ is consistent with the formation of bulk-like clusters at a coverage of 4.60 ML. Likewise, on hBN/ $\mathrm{Cu}(111)$, we observed the $\mathrm{Y}$ and $\mathrm{Y}$ ' lines for coverages from about 4-5 ML onward (see Section 3.1) in parallel with $\mathrm{FL}_{\mathrm{A}}$, $\mathrm{FL}_{\mathrm{B}}$, and $\mathrm{FL}_{\mathrm{C}}$. This confirms, in accordance with our LEED data, that at least two complete layers of PTCDA form on both surfaces under the given preparation conditions.

\section{3 $\mathrm{FL}$ at defect positions}

As stated above, we assign $\mathrm{FL}_{\mathrm{A}}$ to molecules adsorbed at surface defects. Since $\mathrm{FL}_{\mathrm{A}}$ can be observed for PTCDA/hBN/ $\mathrm{Cu}(111)$ and PTCDA/Cu(111), we assume the same kind of defects has to be present on both surfaces. We propose that these defects are different to the metallic "hot spots" supporting SERS because $\mathrm{FL}_{\mathrm{A}}$ is shifted in energy, which is less expected for coupling to metallic protrusions. An alternative origin of these defects could be the presence of carbon containing species due to segregation from the $\mathrm{Cu}$ bulk. Although this interpretation is speculative, we give some background in order to aid subsequent research. When the $\mathrm{Cu}(111)$ crystal was annealed at a temperature above $1050 \mathrm{~K}$ and for a period of time longer than 30 min, we observed an additional superstructure in LEED. Although we cannot identify these segregations unambiguously, we assume carbon segregation as carbon is known to form highly ordered nanostructures on $\mathrm{Cu}(111)$ [69]. Indeed, we adjusted our preparation by reducing the temperature to $1010 \mathrm{~K}$ during borazine deposition and omitting the annealing step under vacuum before borazine deposition to avoid this segregation (see Experimental section). However, we cannot exclude a small and randomly distributed residual amount of segregated carbon species since these would be invisible in LEED. We indeed found that samples showing segregated carbon in LEED do not allow for the formation of the hBN layer. Presumably, due to the extended carbon coverage, there is not enough bare $\mathrm{Cu}$ surface left for supporting the catalytic growth of hBN. Ac- 
cordingly, on a surface with a small amount of carbon, growth defects in the hBN layer at positions where carbon is present may be expected. Thus, we consider carbon species in direct contact with the bare $\mathrm{Cu}$ that lead to similar or even identical defects on both surfaces (hBN/Cu(111) and bare $\mathrm{Cu}(111)$ ), which, in turn, promote identical FL signals of PTCDA molecules. Note that these carbon-related defects are different from the defects that cause the SERS effect. The hot spots that lead to an enhancement of Raman signals are caused by structural defects of the $\mathrm{Cu}$ crystal, such as surface roughness, and not by a different chemical species.

Besides $\mathrm{FL}_{\mathrm{A}}$, no further FL can be observed for $1.55 \mathrm{ML}$ PTCDA/Cu(111) despite the fact that the LEED pattern shows well-resolved spots of ordered domains. The absence of FL from these ordered domains indicates that a single PTCDA monolayer on $\mathrm{Cu}(111)$ cannot decouple PTCDA in the second layer from the metal surface sufficiently enough for FL to occur. Thus, the observation of only $\mathrm{FL}_{\mathrm{A}}$ for a coverage of 1.55 ML leads to the conclusion that the defects at which the fluorescing molecules are adsorbed also have a decoupling effect. This corroborates our deduction that the defects cannot be $\mathrm{Cu}$ ad-atoms.

We would like to reiterate that we exclude the possibility that the $\mathrm{FL}_{\mathrm{A}}$ of PTCDA on $\mathrm{hBN} / \mathrm{Cu}(111)$ stems from molecules trapped in the "moirons" of the hBN layer [24]. Studies on $\mathrm{hBN} / \mathrm{Cu}(111)$ have shown a trapping of large organic molecules in the moirons of the electronic superstructure of hBN/ $\mathrm{Cu}(111)$ at low molecular coverages [38]. At larger coverages, the entire surface was found to be homogeneously filled with molecules. Yet, they still showed site-dependent alterations in their electronic structure, namely a shift of the molecular frontier orbitals [38]. However, we propose that the situation of PTCDA on hBN/Cu(111) is different. First of all, as stated above, $\mathrm{FL}_{\mathrm{A}}$ is also observed for PTCDA on $\mathrm{Cu}(111)$, which clearly excludes trapped PTCDA molecules on hBN as a possible origin for $\mathrm{FL}_{\mathrm{A}}$. Furthermore, our own STM investigations showed no signs of a preferential occupation of the moirons on the hBN/Cu(111) surface by PTCDA [32]. Also, holes in the $\mathrm{hBN}$ layer that would allow for a direct contact between the molecules and the bare $\mathrm{Cu}(111)$ surface can be excluded as the origin for $\mathrm{FL}_{\mathrm{A}}$ because the FL of PTCDA molecules in direct contact with the metal would be fully quenched.

\section{Final discussion}

We found that two layers of PTCDA are necessary to decouple PTCDA molecules from the $\mathrm{Cu}(111)$ surface in order to observe FL from ordered domains in the third layer. The same effect can be achieved by only one single layer of $\mathrm{hBN}$. On $\mathrm{hBN} / \mathrm{Cu}(111)$, two FL components $\left(\mathrm{FL}_{\mathrm{A}}\right.$ and $\left.\mathrm{FL}_{\mathrm{B}}\right)$ are present for the first
PTCDA layer, while a third one $\left(\mathrm{FL}_{\mathrm{C}}\right)$ can only be observed from the second layer onward. On $\mathrm{Cu}(111)$, the FL from the first PTCDA layer is completely quenched. $\mathrm{FL}_{\mathrm{A}}$ and $\mathrm{FL}_{\mathrm{C}}$ are observed only from the second and third layer onward, respectively. $\mathrm{FL}_{\mathrm{A}}$ is assigned to a defect-related $\mathrm{FL}$, while both $\mathrm{FL}_{\mathrm{B}}$ and $\mathrm{FL}_{\mathrm{C}}$ are assigned to ordered PTCDA domains. We can exclude bulk-like crystallites on top of the monolayer as the origin of the $\mathrm{FL}_{\mathrm{B}}$ and $\mathrm{FL}_{\mathrm{C}}$ peaks by the following arguments: (i) The LEED patterns of PTCDA on $\mathrm{hBN} / \mathrm{Cu}(111)$ and on $\mathrm{Cu}(111)$ identify the formation of long-range ordered structures in the second layer. (ii) LEED investigations (see Appendix A) and TPD experiments $[32,68]$ show that PTCDA/Cu(111) and $\mathrm{PTCDA} / \mathrm{hBN} / \mathrm{Cu}(111)$ form at least three or two complete layers, respectively. (iii) The spectral positions of $\mathrm{FL}_{\mathrm{B}}$ and $\mathrm{FL}_{\mathrm{C}}$ are unambiguously distinct from the $\mathrm{Y}$ and $\mathrm{Y}^{\prime}$ peaks of the FL of the PTCDA bulk.

We return to the question of CT across the hBN layer posed in the Introduction section. Our results indicate that the hBN layer is able to suppress the CT such that FL is observed. However, this suppression is not complete because the FL intensity is very low. In comparison to the FL of PTCDA observed on thin $\mathrm{KCl}$ films, we reckon that the $\mathrm{FL}$ on $\mathrm{hBN} / \mathrm{Cu}(111)$ is smaller by a factor of ca. $10^{4}$ [70]. However, hBN should not be dismissed as a decoupling layer altogether. In an analogue experiment, we measured the FL spectrum of $5,10,15,20$ tetraphenylbisbenz[5,6]indeno[1,2,3-cd:1',2',3'-lm]perylene (DBP) on $\mathrm{hBN} / \mathrm{Cu}(111)$ (not shown). (For the chemical formula and optical properties of DBP, refer to the work by Rouillé et al. [71].) Here, we observed FL at sub-monolayer coverage [72]. The FL intensity was larger by a factor of ca. $10^{2}$ compared to the FL intensity of PTCDA on $\mathrm{hBN} / \mathrm{Cu}(111)$. This can be explained by the fact that DBP is a lander-type molecule. It exhibits four peripheral phenyl groups, which function as spacers between the molecular backbone and the surface [71]. This presumably, in addition to the hBN layer and a weak interaction, supports the suppression of the CT process and allows for a higher FL intensity.

We further discuss the widths of the FL peaks on $\mathrm{Cu}(111)$ and $\mathrm{hBN} / \mathrm{Cu}(111)$ in some detail. The large width of the FL peaks may partly be caused by disorder in the molecular domains or by a very small lifetime of the excited state. Since our LEED experiments showed the formation of ordered PTCDA domains, we assume that the main contribution for the line broadening is due to a reduced FL lifetime, $\tau_{\mathrm{FL}}$, and that contributions from disorder and dephasing can be neglected $[73,74]$. Thus, the value of $\tau_{\mathrm{FL}}$ measured from the full width at half maximum (FWHM) of the FL peaks on the frequency scale according to $\mathrm{FWHM}=1 / 2 \pi \tau_{\mathrm{FL}}$ yields a lower limit for the lifetime of the excited states. Because of the small FL yield we can assign $\tau_{\mathrm{FL}}$ 
in good order to the time constant of the $\mathrm{CT}$ process. Hence, $\tau_{\mathrm{FL}}$ gives quantitative information about the efficiency of the decoupling from the metal interface. Figure 6 representatively shows region II of the optical spectra of $0.60 \mathrm{ML}$ PTCDA/hBN/ $\mathrm{Cu}(111)$ and $2.55 \mathrm{ML}$ PTCDA/Cu(111). Both spectra show $\mathrm{FL}_{\mathrm{A}}$ and $\mathrm{FL}$ from ordered domains as either $\mathrm{FL}_{\mathrm{B}}$ or $\mathrm{FL}_{\mathrm{C}}$, respectively. The FL peaks and the Raman modes were fitted with sets of Lorentzians. The widths and positions of the narrower Raman lines were fixed during the fits. The fits were thus robust concerning the determination of the FWHM of the FL peaks. The lifetimes derived from these fits are given in Table 3. All of

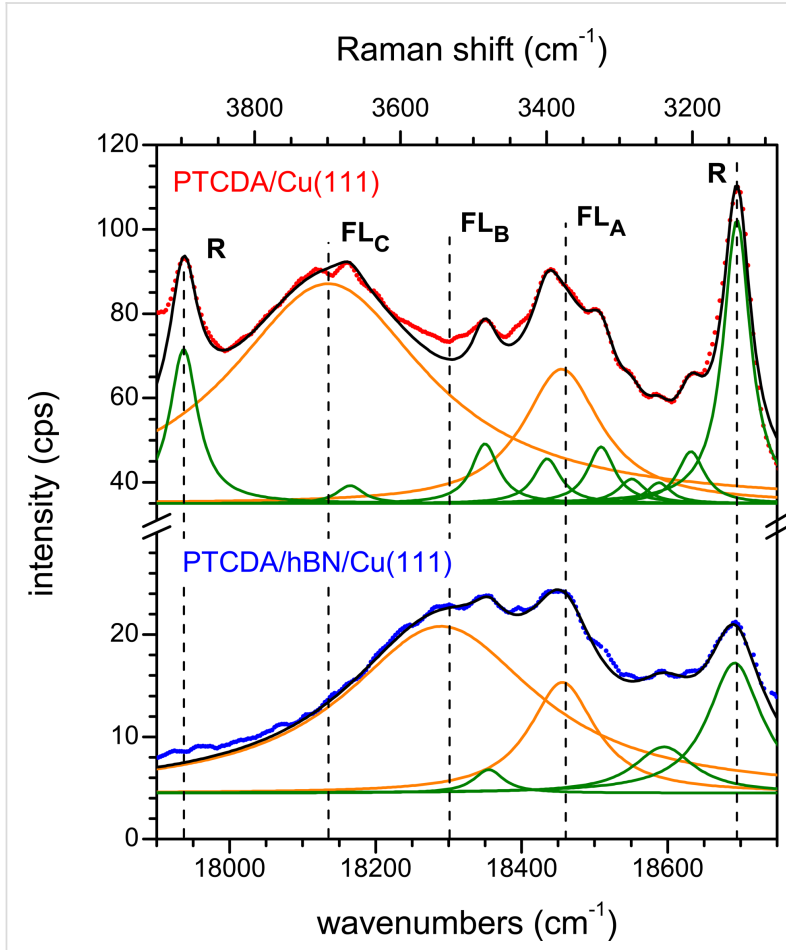

Figure 6: Fluorescence spectra of $0.60 \mathrm{ML}$ PTCDA/hBN/Cu(111) (blue, bottom) and 2.55 ML PTCDA/Cu(111) (red, top). Original data (dots) are smoothed and shifted vertically. Both spectra were fitted using Lorentzian functions for the FL peaks (orange lines) and the most important Raman modes (green lines). Cumulative fits shown in black. Spectra of the clean substrates were subtracted as a background (cf. Figure 5). All spectra were measured at $20 \mathrm{~K}$ using a grating with 600 grooves per millimeter. them are of a similar size and of the order of $10^{-14} \mathrm{~s}$. Thus, we can understand why we did not observe final-state effects in the photoemission spectra (i.e., UPS) of PTCDA/hBN/Cu(111) due to $\mathrm{CT}$ across the interface, that is, the signature of uncoupled molecules [32], albeit the FL experiments demonstrate that some coupling to the underlying $\mathrm{Cu}(111)$ surface is present. The reason is that the time constant of this coupling is only of the same order as the time constant of the photoemission process. Therefore, it has only a small or negligible impact on the spectra. These small lifetimes are in agreement with the findings by Stallberg et al. [39], who showed that the lifetimes of the excited states of PTCDA in the second layer on $\mathrm{Ag}(111)$ and in the third layer on $\mathrm{Au}(111)$ are smaller than an upper value of $4 \times 10^{-12} \mathrm{~s}$, which was given by the time resolution for their experiment. The small lifetimes also explain why the FL intensities were so low with respect to those measured, for example, for an ordered monolayer of PTCDA/KCl/Ag(100) [65].

Both the small FL intensities and the short lifetime of the excited state of PTCDA on $\mathrm{hBN} / \mathrm{Cu}(111)$ show that, at least for this molecule, a single hBN layer is not sufficient to completely decouple the electronic states of the molecule and the metal. While the static CT related to the interfacial bonding is suppressed, as demonstrated by UPS [32], it is still significant for the excited state. A similar observation has been made for tetracene molecules on thin insulating layers of $\mathrm{AlO}_{x}$ on $\mathrm{Ni}_{3} \mathrm{Al}(111)$ [75]. Here, too, the luminescence was found to be quenched despite the large bandgap of $\mathrm{AlO}_{x}(6.5 \mathrm{eV})$ and the weak interactions at the interface due to a CT between overlapping $\pi$ orbitals and the electronic states of the metal.

\section{Conclusion}

We have measured the fluorescence of PTCDA on hBN/ $\mathrm{Cu}(111)$ and $\mathrm{Cu}(111)$ to determine the efficiency of the electronic decoupling of PTCDA from the $\mathrm{Cu}$ substrate by a single hBN layer. The observation of Raman lines served as a monitor for the presence of PTCDA on the surface. In addition, LEED patterns show the formation of ordered structures and a layerby-layer growth for at least the first two layers on $\mathrm{hBN} / \mathrm{Cu}(111)$ and the first three layers on $\mathrm{Cu}(111)$.

Table 3: Spectral positions of the FL peaks observed for PTCDA/hBN/Cu(111) and PTCDA/Cu(111), the species the FL peaks originate from, the FWHM of the FL peaks, and the lifetimes $T_{F L}$ of the respective excited states. Defect FL and physisorbed PTCDA apply for both PTCDA/hBN/Cu(111) and PTCDA/Cu(111).

\begin{tabular}{llll} 
& $\mathrm{FL}_{\mathrm{A}}$ & $\mathrm{FL}$ & $\mathrm{FL}_{\mathrm{C}}$ \\
\hline FL peak position & $18,450 \mathrm{~cm}^{-1}$ & $18,300 \mathrm{~cm}^{-1}$ & $18,150 \mathrm{~cm}^{-1}$ \\
assignment & defect related FL & $\mathrm{PTCDA} / \mathrm{hBN}$ & physisorbed PTCDA \\
FWHM & $(120 \pm 10) \mathrm{cm}^{-1}$ & $(320 \pm 16) \mathrm{cm}^{-1}$ & $(330 \pm 15) \mathrm{cm}^{-1}$ \\
$\mathrm{~T}_{\mathrm{FL}}$ & $(4.4 \pm 0.3) \times 10^{-14} \mathrm{~s}$ & $(1.7 \pm 0.1) \times 10^{-14} \mathrm{~s}$ & $(1.6 \pm 0.1) \times 10^{-14} \mathrm{~s}$
\end{tabular}


The intensities of the Raman lines do not scale with the coverage, neither on $\mathrm{hBN} / \mathrm{Cu}(111)$, nor on $\mathrm{Cu}(111)$. This clearly shows that additional aspects of the sample system, likely defects of the $\mathrm{Cu}$ substrate, play a role. We summarize the underlying mechanisms under the SERS effect. The small but discernibly different chemical shifts of Raman modes on the two surfaces are explained by the molecule-substrate interactions and specific bonding geometries of the molecule to the surface.

On both substrates, a broad fluorescence at ca. $18,450 \mathrm{~cm}^{-1}$ can be observed, which is identified as FL from molecules interacting with surface defects. This can be observed both for the first PTCDA layer on $\mathrm{hBN} / \mathrm{Cu}(111)$ and the second layer of PTCDA on $\mathrm{Cu}(111)$. FL from the first PTCDA layer on $\mathrm{Cu}(111)$ is not observed. In contrast, the first PTCDA layer on $\mathrm{hBN} / \mathrm{Cu}(111)$ already shows a weak FL from ordered domains at ca. $18,300 \mathrm{~cm}^{-1}$.

On bare $\mathrm{Cu}(111)$, two interfacial layers of PTCDA are required to achieve an equivalent decoupling of the third layer as one layer of $\mathrm{hBN}$ on $\mathrm{Cu}(111)$ for the first PTCDA layer. The third layer on $\mathrm{Cu}(111)$ shows FL at ca. $18,150 \mathrm{~cm}^{-1}$. This FL can be also observed in the second PTCDA layer on $\mathrm{hBN} / \mathrm{Cu}(111)$ and is attributed in both cases to domains of physisorbed PTCDA on a separating PTCDA layer that forms on either the hBN layer or the first chemisorbed interfacial PTCDA monolayer on $\mathrm{Cu}(111)$.

While the charge transfer between PTCDA molecules and metal is sufficiently suppressed by a single $\mathrm{hBN}$ layer such that a weak fluorescence is observed, this suppression is limited. We estimate a lifetime of the excited state of ca. $10^{-14} \mathrm{~s}$, which explains the very small fluorescence intensities. As a result, we find that a single layer of $\mathrm{hBN}$ on $\mathrm{Cu}(111)$ is able to decouple PTCDA molecules from the metal surface in so far as to prevent a total quenching of the fluorescence of the first molecular layer. However, a competing non-radiative channel for the decay of the excited state of the molecule remains as some charge transfer with a time constant of $10^{-14} \mathrm{~s}^{-1}$ is still possible. Thus, while a single layer of $\mathrm{hBN}$ provides a more efficient decoupling of a PTCDA layer from the $\mathrm{Cu}(111)$ surface than the first PTCDA layer itself, its efficiency is limited.

\section{Appendix A: Structural Investigations}

Here, we present the structural investigations of monolayer PTCDA on $\mathrm{hBN} / \mathrm{Cu}(111)$ and $\mathrm{Cu}(111)$ by LEED.

\section{The structure of PTCDA/Cu(111)}

We begin with a summary of earlier reported results. At RT, PTCDA on the $\mathrm{Cu}(111)$ surface follows a layer-by-layer growth mode for the first three layers [76]. The obtained films remain unchanged under annealing at $530 \mathrm{~K}$. From the fourth layer onward, the formation of nanocrystals begins. In TPD spectra, the desorption of the multilayer can be distinguished from the desorption of the second layer [68]. A desorption of the first layer was not observed due to a chemisorptive bonding to the $\mathrm{Cu}(111)$ surface. An STM study on the PTCDA monolayer on $\mathrm{Cu}(111)$ was published by Wagner et al. [77]. The authors reported two co-existing PTCDA phases, both displaying the characteristic herringbone arrangement, one commensurate, the other commensurate only in higher order.

In our experiments, PTCDA layers on $\mathrm{Cu}(111)$ were always grown at a sample temperature of $300 \mathrm{~K}$. Experiments conducted in two different UHV chambers with different deposition rates resulted in identical LEED patterns. All LEED measurements were performed at $100 \mathrm{~K}$.

Figure 7a shows the LEED pattern of a complete monolayer PTCDA/Cu(111) with sharp diffraction spots. The spots marked in dark red are explained by one single phase $(\alpha)$. Additionally, a second phase ( $\beta$, marked in green in Figure 7$)$ is present. From the relative spot intensities, the $\alpha$ phase is considered as the majority phase. The monolayer LEED pattern is fully explained by the $\alpha$ and $\beta$ phases with no systematic differences between the LEED pattern and the simulation. There are systematic extinctions of all $\{h 0\}$ and $\{0 k\}$ spots for which $h$ and $k$ are odd. This points to the presence of glide planes in both phases. The structural parameters, which can be determined from the LEED pattern with small margins of error (ca. 5\%), are given in Table 4. The first columns of the superstructure matrices of the $\alpha$ phase and the $\beta$ phase exhibit elements that are integer numbers (within the margins of error). This means that both phases exhibit on-line-coincidence (OLC) [78] with the $\mathrm{Cu}(111)$ surface.

Both phases ( $\alpha$ and $\beta$ ) exhibit a rectangular unit cell and belong to the $p 2 g g$ space group. The differences between the two are the azimuthal orientations of the unit cell vectors relative to the $\mathrm{Cu}(111)$ surface vectors and the packing densities. The unit cell of the $\alpha$ phase is more closely aligned with the $\mathrm{Cu}$ surface vector $\mathbf{a}_{1}\left(2.5^{\circ}\right)$ compared to the $\beta$ phase $\left(10.3^{\circ}\right)$. Furthermore, the molecules in the $\beta$ phase are slightly more densely packed than in the $\alpha$ phase $\left(A_{\alpha}=103 \% \times A_{\beta}\right.$, cf. Table 4$)$.

The most prominent feature of the LEED pattern is the characteristic arrangement of spots forming a "double triangle" (marked by a red box in Figure 7a). This is well known for the rectangular unit cell of PTCDA molecules forming the typical herringbone motif $[80,81]$ that can also be found in the (102) plane of the bulk material [82]. In contrast to other coinage 


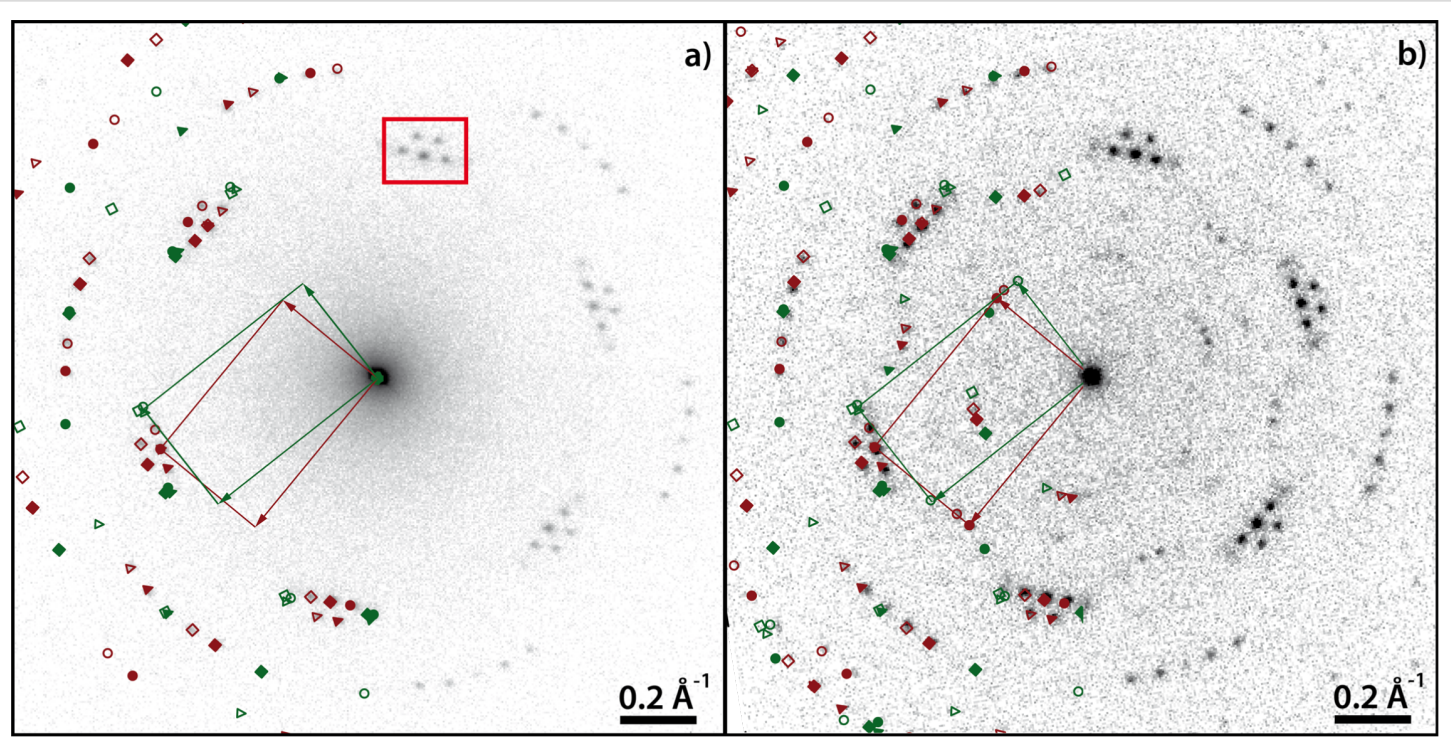

Figure 7: LEED pattern of (a) $1 \mathrm{ML}$ PTCDA/Cu(111) and (b) $1.55 \mathrm{ML}$ PTCDA/Cu(111) deposited at $300 \mathrm{~K}$. On the left-hand side, the LEED patterns are superimposed with the corresponding simulations. Different colors refer to different PTCDA phases (dark red: $\alpha$ phase, green: $\beta$ phase) and different symbols refer to different symmetry equivalent domains of a specific phase. The LEED patterns differ in the diffraction spots $\{h 0\}$ and $\{0 k\}$ for which $h$ and $k$ are odd. They are present in (b), but extinct in (a). The red box in (a) highlights the characteristic double-triangle arrangement of spots of the herringbone structure of PTCDA on surfaces. The LEED patterns were recorded at an electron energy of $77.6 \mathrm{eV}$ and at a sample temperature of $100 \mathrm{~K}$.

Table 4: Structural results of PTCDA/Cu(111) according to the structure model proposed by Wagner et al. [77] (phase '1' and '2') and as determined from our LEED experiments ( $\alpha$ phase and $\beta$ phase) and of PTCDA/hBN/Cu(111). The vectors of the unit cells, $\mathbf{b}_{1}$ and $\mathbf{b}_{2}$, the angle $\beta$ between the two, and the areas of the unit cells, $A$, are given. $\phi$ is the enclosed angle between the vector $\mathbf{b}_{1}$ and the substrate lattice vector, $\mathbf{a}_{1} . \rho$ is the packing density of the molecules. Additionally, the superstructure matrices, M, are given. The matrix for PTCDA/hBN/Cu(111) refers to the Cu(111) surface at $100 \mathrm{~K}$. We note that the parameters of phase '1' and ' 2 ' are derived from the (higher-order) commensurate structure models given in [77] and slightly deviate from the experimental STM results (within error margins). The error for $\beta$ is the experimental error of the STM data. The matrices given for phase ' 1 ' and ' 2 ' differ from those reported by Wagner et al. [77] because they refer to a symmetry-equivalent domain according to the rules given in [79].

$$
\text { Cu(111) - phase '1' [77] Cu(111) - phase '2' [77] }
$$

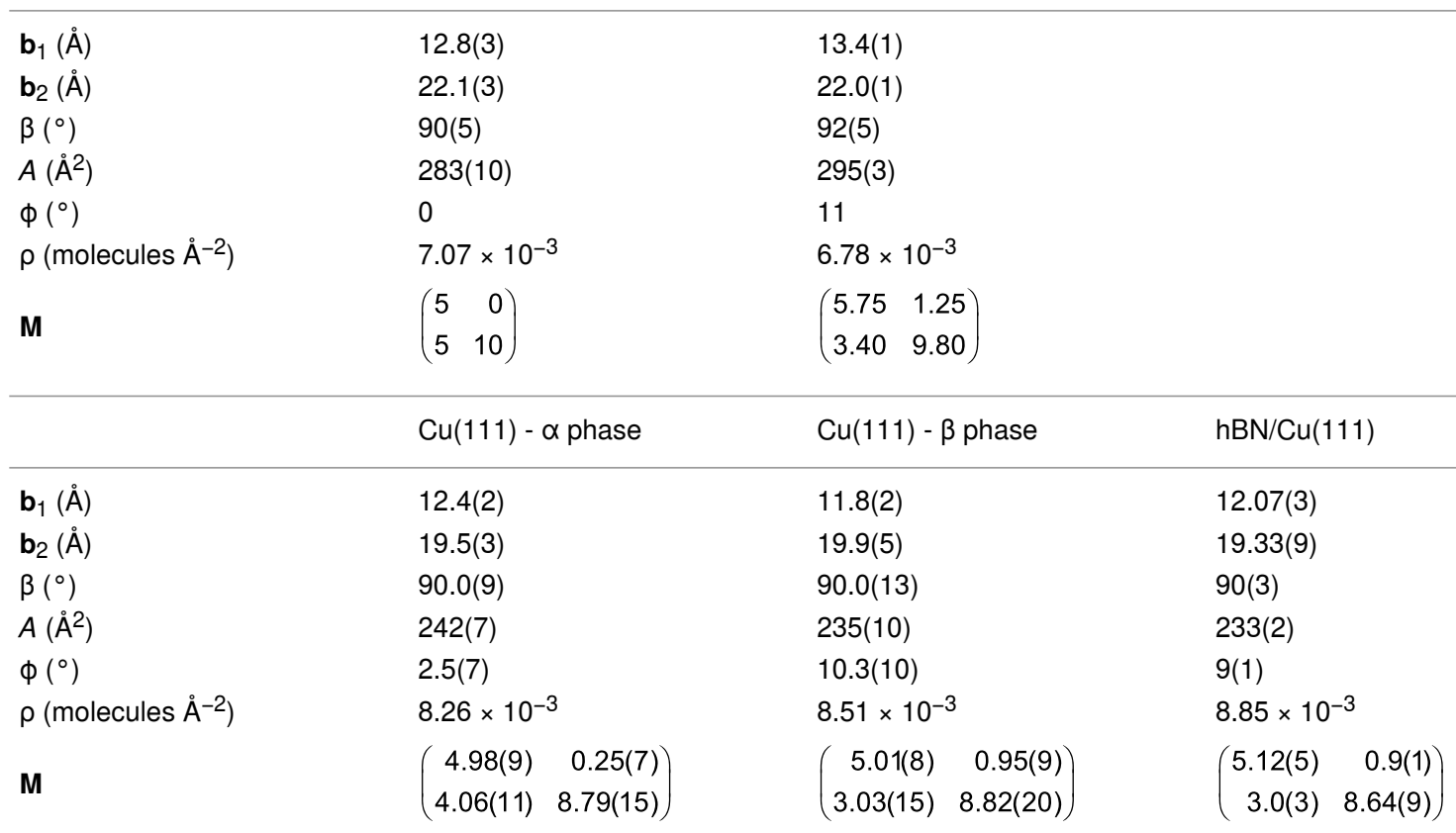


metal substrates, however, where two separate triangles consisting of six diffraction spots are observed [80,83]. Here, the $\{11\}$ and $\{20\}$ spots coincide, leading to two triangles that are characteristically corner-connected by one spot.

The PTCDA $\beta$ phase on $\mathrm{Cu}(111)$ found in LEED bears significant similarity to the structure ' 2 ' proposed by Wagner and co-workers [77]. Using the same rules for the matrix notations [79] for all structures shows that the matrix elements of structure ' 2 ' and of the $\beta$ phase found in our work differ only slightly (only one entry differs by more than $13 \%$ ). The orientations of the unit cell vector $\mathbf{b}_{1}$ with respect to the substrate is identical for both structures within $\pm 1.6^{\circ}$ (cf. Table 4 ). Hence, we propose that the $\beta$ phase possibly corresponds to structure ' 2 ' by Wagner, although a difference in the size of the unit cell remains. Structure ' 1 ' given by Wagner, however, was not observed in our LEED experiments and differs from our $\alpha$ phase. Furthermore, the packing densities found for the $\alpha$ phase and the $\beta$ phase agree well (within $2.5 \%$ ) with those of the (102) planes of the PTCDA bulk phases $\left(8.40 \times 10^{-3}\right.$ and $8.32 \times 10^{-3}$ molecules $\AA^{-2}$, respectively [82]), while the densities in structures ' 1 ' and ' 2 ' differ more significantly from these (by $14 \%$ and $21 \%$ ). These differences may possibly be due to a difference in the substrate quality or preparation conditions in the two studies.

We now turn to PTCDA coverages above $1 \mathrm{ML}$. Both monolayer phases of PTCDA on $\mathrm{Cu}(111)(\alpha$ and $\beta$ ) exist in parallel from the (sub-)monolayer to the multilayer regime. Additional deposition of PTCDA up to a coverage of 3 ML leads to additional weak spots in the LEED pattern (see Figure 7b). They can also be explained by the two PTCDA phases identified above. The spots that appear at distances of $0.3-0.6 \AA^{-1}$ from the specular spot correspond to $\{h 0\}$ and $\{0 k\}$ spots of the $\alpha$ phase and the $\beta$ phase for which $h$ and $k$ are odd and which were extinct before. No spot shifts as a function of the coverage were found. This may have two reasons. Either the molecules form only a single wetting layer on which additional molecules form clusters, or the higher layers adapt the same structure as the first layer without mismatch. Two observations point to the latter case: The intensity of the adsorbate diffraction spots increases with coverage while the relative intensity of the specular spot decreases. In addition, the $\{10\}$ and $\{01\}$ spots become detectable, which means that the glide planes of the adsorbate structure vanish. The glide lines in the space group $p 2 g g$ of the PTCDA unit cell only exist if the underlying $\mathrm{Cu}(111)$ surface is not taken into account. For the first monolayer, this may be the case because the small periodicity of the $\mathrm{Cu}$ atoms compared to the PTCDA periodicity is not "felt" by the electrons scattered by a single PTCDA layer. For a second PTCDA layer, however, the periodicities in this layer and in the underlying monolayer are identical. Both layers are shifted laterally against each other [82], which breaks the glide plane symmetry. Thus, the LEED data indicate the growth of at least two or even more complete layers of PTCDA on $\mathrm{Cu}(111)$. This is in accordance with the results reported in $[68,76]$.

In summary, our LEED investigations show that PTCDA on $\mathrm{Cu}(111)$ forms two co-existing phases, both of them displaying on-line-coincidence to the surface with a herringbone arrangement of the molecules. Both are observed from the (sub-)monolayer regime to at least the beginning of the growth of the third layer. The adsorbate forms at least two complete layers before clusters form.

\section{PTCDA on $\mathrm{hBN} / \mathrm{Cu}(111)$}

Figure 8 shows LEED patterns of PTCDA/hBN/Cu(111) at a coverage of $2 \pm 0.5 \mathrm{ML}$ (Figure 8a,c) and $0.8 \pm 0.2 \mathrm{ML}$ (Figure $8 \mathrm{~b}$ ). All structures were grown at a sample temperature of $260 \mathrm{~K}$ and a deposition rate of $1 \mathrm{ML} / \mathrm{min}$. They were observed in two different UHV chambers. LEED measurements were performed at $110 \mathrm{~K}$. Note that the LEED pattern in Figure 8a has previously been published in [32]. In that study, we attributed this LEED pattern to a pure monolayer of PTCDA on $\mathrm{hBN} / \mathrm{Cu}(111)$. Further analysis now leads us to the conclusion that the LEED pattern stems from a slightly higher coverage. This will be discussed in further detail below. Nonetheless, our findings presented in [32] still hold.

Commonly, two kinds of LEED patterns were observed for $\mathrm{PTCDA} / \mathrm{hBN} / \mathrm{Cu}(111)$ and were found from the sub-monolayer to the multilayer regime. The observable pattern, and thus the PTCDA structure that formed, did not depend on the PTCDA coverage, but rather on the quality of the hBN layer. The azimuthally smeared out intensity in Figure 8b,c (which was most commonly observed) points to an azimuthal disorder of the PTCDA domains on $\mathrm{hBN} / \mathrm{Cu}(111)$, both in the first and in higher layers. In some cases, LEED patterns such as that given in Figure 8a were observed, which shows spots that are exceptionally not smeared out azimuthally. The radii of the rings in Figure 8b,c match the distances of the spots from the specular spot in Figure 8a.

We attribute the higher azimuthal order, which manifests in the more discrete spots, to a lower-quality hBN layer with defects or uncovered areas of $\mathrm{Cu}(111)$. Both may allow a pinning of PTCDA molecules to the $\mathrm{Cu}(111)$ surface. These molecules function as growth nuclei for PTCDA domains and determine the azimuthal orientation of these domains. Evidence for this interpretation is given by the fact that the orientation of the PTCDA domains on $\mathrm{hBN} / \mathrm{Cu}(111)$ is in agreement with the orientation of PTCDA domains in the $\beta$ phase on $\mathrm{Cu}(111)$ (within 


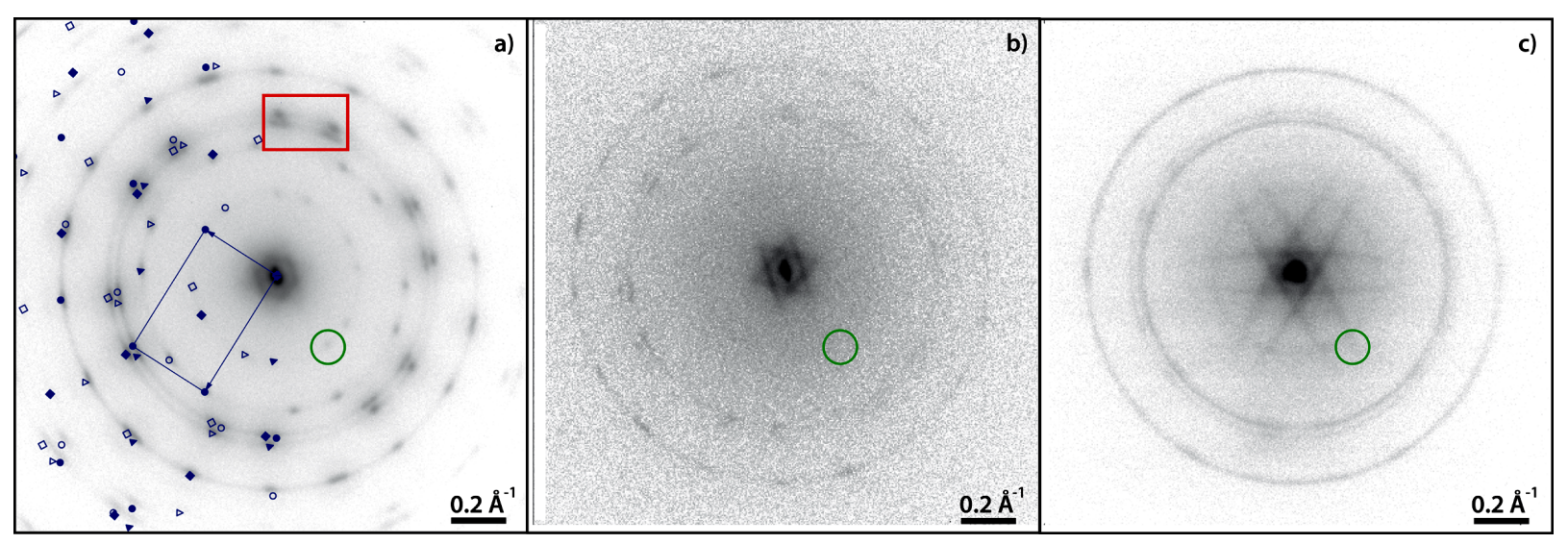

Figure 8: LEED pattern of PTCDA layers on $h B N / C u(111)$. (a) LEED pattern of $2 \pm 0.5 \mathrm{ML}$ PTCDA/hBN/Cu(111). The intensities of the LEED spots are azimuthally smeared out. The maxima in the ring intensities indicate the presence of azimuthally ordered domains. The left half of the LEED pattern is superimposed with the corresponding simulation. Different symbols refer to different symmetrically equivalent domains. This is the same LEED pattern as the one shown in [32], which we originally interpreted as a monolayer. More detailed analysis shows now that the second PTCDA layer has started to form (see main text). The red box highlights the characteristic double-triangle arrangement of spots of the herringbone structure of PTCDA on surfaces. The green ring marks a $\{10\}$ diffraction spot. (b) LEED pattern of $0.8 \pm 0.2 \mathrm{ML}$ PTCDA/hBN/Cu(111). The LEED intensity is homogeneously distributed in rings around the specular spot. The radii of the rings match the distances of the spots in (a) from the specular spot. The green ring marks the same position as in (a). However, the rings corresponding to spot positions of $\{h 0\}$ and $\{0 k\}$ for which $h$ or $k$ are odd are extinct. (c) LEED pattern of $2 \pm 0.5 \mathrm{ML}$ PTCDA/hBN/Cu(111). The LEED intensity is homogeneously distributed in rings around the specular spot as in (b). At this higher coverage, the first-order PTCDA spots are no longer extinct. At the position of the green circle, a faint homogeneous ring can hence be observed. All LEED patterns were recorded at an electron energy of $31 \pm 1 \mathrm{eV}$ and at a sample temperature of $110 \mathrm{~K}$.

the error margins, cf. Table 4). The comparison to PTCDA/ $\mathrm{Cu}(111)$ shows that the $\beta$ phase has a higher structural resemblance with PTCDA/hBN/Cu(111) than the majority $\alpha$ phase, not only with regard to the orientation, but also to the size of the unit cell (with a deviation of $0.85 \%$ of the areas of the unit cells of PTCDA/hBN/Cu(111) and the $\beta$ phase).

However, in general, PTCDA domains on $\mathrm{hBN} / \mathrm{Cu}(111)$ have no intrinsically preferred orientation. In Figure $8 \mathrm{~b}$ and Figure $8 \mathrm{c}$, near the specular spot, a star-like pattern caused by multiple scattering of electrons on $\mathrm{hBN} / \mathrm{Cu}(111)$ [30] can be observed. Its sharpness points to the high quality of the $\mathrm{hBN}$ layer that leads to the homogeneous distribution of the azimuthal orientation of the PTCDA domains.

The discrete spots in Figure 8a support the determination of the structural parameters (cf. Table 4). The similarity of this LEED pattern to those of other PTCDA structures on surfaces is obvious. This includes the double-triangle arrangement of spots (marked by a red box), which makes the structural difference of PTCDA on $\mathrm{Cu}(111)$ and on $\mathrm{hBN} / \mathrm{Cu}(111)$ apparent. Here, the double triangle is formed by six spots leading to two separated triangles. (On $\mathrm{Cu}(111)$, these are connected at their corners.) The structure is incommensurate to the $\mathrm{hBN}$ layer, and also to the underlying $\mathrm{Cu}(111)$ surface. This is in accordance with a weak corrugation of the PTCDA/hBN interaction potential. The
LEED pattern points to an arrangement of the molecules in the herringbone motif, as on $\mathrm{Cu}(111)$ [77] and other substrates [80,83]. This was also seen in STM measurements [32].

A comparison between the LEED pattern of the first PTCDA layer on $\mathrm{hBN} / \mathrm{Cu}(111)$ (Figure $8 \mathrm{~b}$ ) with those of higher PTCDA layers (Figure 8a,c) shows that the diffraction spots $\{h 0\}$ and $\{0 k\}$ for which $h$ or $k$ are odd are extinct for the monolayer, but appear in the second layer. In Figure $8 \mathrm{a}$, a discrete $\{10\}$ spot is marked by a green circle. In Figure $8 \mathrm{c}$, a line of a continuous ring that corresponds to the $\{10\}$ spots of PTCDA is seen in the same circle. In Figure 8b, however, there is no intensity at this position in the LEED pattern. The same discussion on the extinction as for PTCDA/Cu(111) applies here (see Section "The structure of PTCDA/Cu(111)"). Furthermore, the distance of the diffraction spots from the specular spot does not change with increasing coverage. This indicates the same layer-by-layer growth mode of PTCDA on both $\mathrm{Cu}(111)$ and $\mathrm{hBN} / \mathrm{Cu}(111)$.

In summary, monolayers of PTCDA/Cu(111) and PTCDA/ $\mathrm{hBN} / \mathrm{Cu}(111)$, despite their structural similarity, can be distinguished by their LEED patterns on the basis of two crucial features. On $\mathrm{Cu}(111)$, the "double triangle" is formed by five spots, making it a corner-connected double triangle, while on $\mathrm{hBN} / \mathrm{Cu}(111)$ six spots form two distinct triangles. Furthermore, the azimuthal smearing of the spots of PTCDA on hBN/ 
$\mathrm{Cu}(111)$ is specific. Additionally, LEED allows the identification of a second PTCDA layer on $\mathrm{Cu}(111)$ and $\mathrm{hBN} / \mathrm{Cu}(111)$ due to the appearance of additional spots, which are absent in the monolayers for symmetry reasons.

\section{Appendix B: Additional optical spectra}

Here, additional optical spectra that were referred to in the main text are shown. As mentioned in Section 3.1, the Raman lines in region III of the optical spectra were identified using a dye laser with a tunable wavelength. The Raman lines shift according to the wavelength of the laser. Figure 9 shows spectra recorded with wavelengths between 497 and $507 \mathrm{~nm}$ as a function of the Raman shift. Here, all Raman peaks stay at the same energies as expected. Notably, the intensities of the Raman modes change as a function of wavelength. The highest intensities are observed for $458 \mathrm{~nm}$, exceeding the intensities for $502.66 \mathrm{~nm}$ (the highest in Figure 9) by a factor of seven.

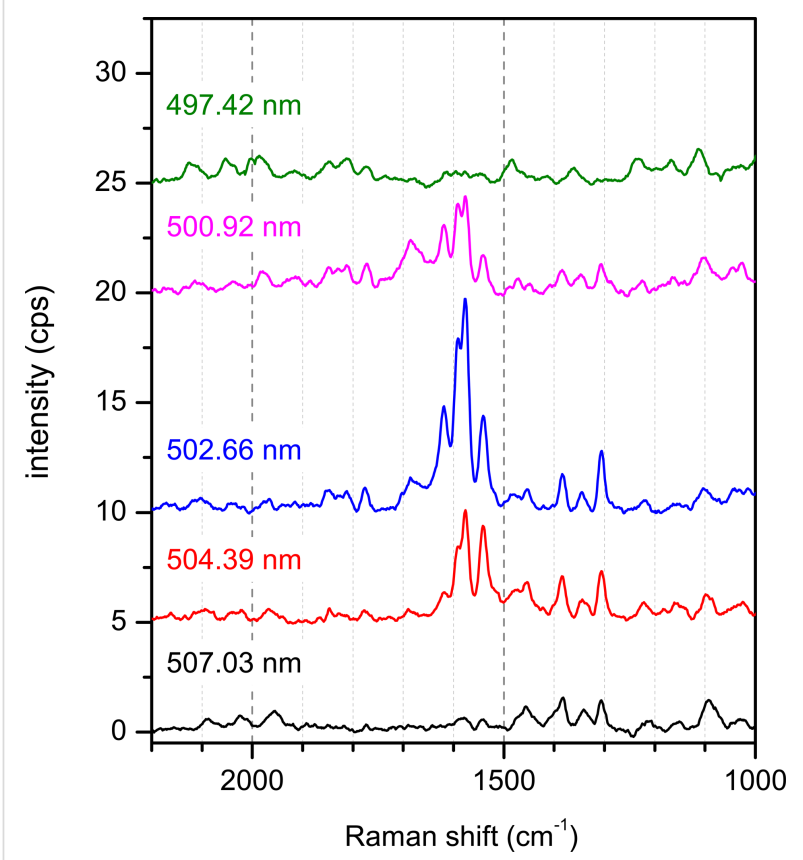

Figure 9: Raman modes of 0.90 ML PTCDA on hBN/Cu(111), measured with a dye laser with tunable wavelength $(497-507 \mathrm{~nm})$. Data are smoothed and shifted vertically after subtraction of a background. All spectra were measured at $20 \mathrm{~K}$ using a grating with 1200 grooves per millimeter.

In Section 3.1, we stated that PTCDA deposited on hBN/ $\mathrm{Cu}(111)$ at a sample temperature of $300 \mathrm{~K}$ leads to the same FL as PTCDA deposited at $20 \mathrm{~K}$ and subsequently annealed at 300 K. Figure 10 shows spectra from PTCDA/hBN/Cu(111) prepared according to either recipe. In both cases, $\mathrm{FL}_{\mathrm{A}}$ and $\mathrm{FL}_{\mathrm{B}}$ are present.

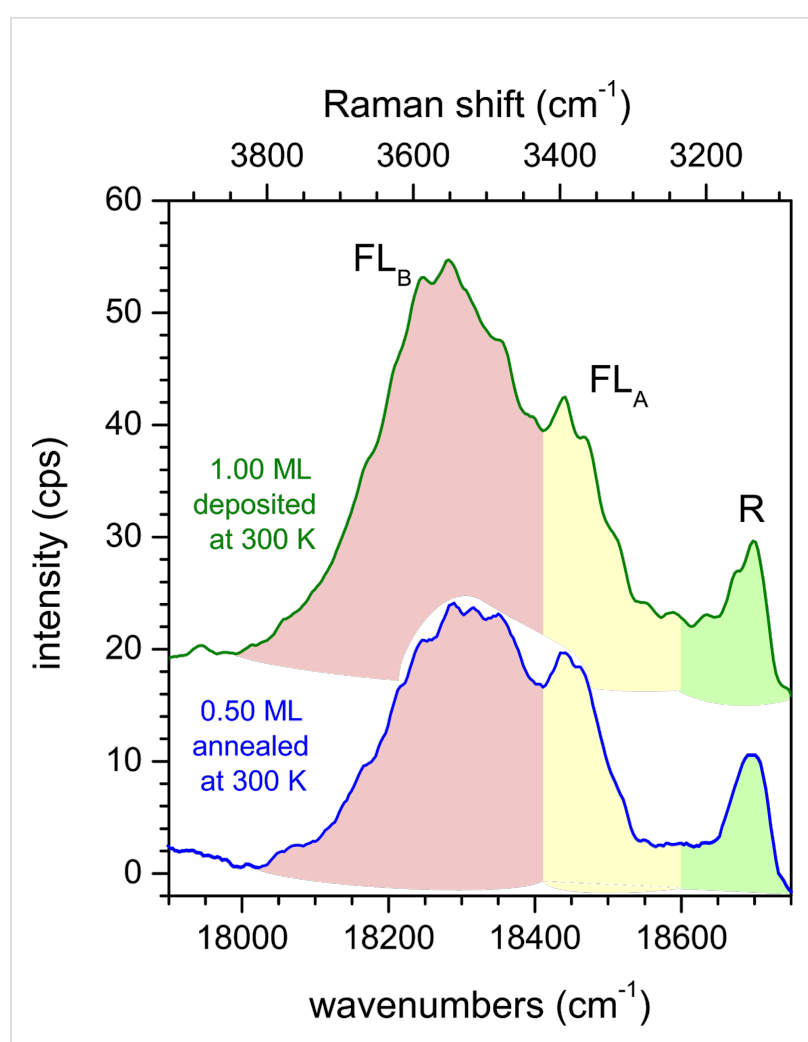

Figure 10: FL spectra of $0.50 \mathrm{ML}$ PTCDA on hBN/Cu(111), deposited at $20 \mathrm{~K}$ and subsequently annealed at $300 \mathrm{~K}$ (blue, c.f Figure 2) and $1.00 \mathrm{ML}$ PTCDA on hBN/Cu(111), deposited at $300 \mathrm{~K}$ (green). Data are smoothed and shifted vertically after subtraction of a background. All spectra were measured at $20 \mathrm{~K}$ using a grating with 600 grooves per millimeter.

\section{Acknowledgements}

We wish to acknowledge C. Marquardt, M. Scheuermann, and B. Wolff for experimental support and E. LeMoal and V. Wagner for helpful discussions.

\section{References}

1. Xu, M.; Liang, T.; Shi, M.; Chen, H. Chem. Rev. 2013, 113, 3766-3798. doi:10.1021/cr300263a

2. Tan, C.; Cao, X.; Wu, X.-J.; He, Q.; Yang, J.; Zhang, X.; Chen, J.; Zhao, W.; Han, S.; Nam, G.-H.; Sindoro, M.; Zhang, H. Chem. Rev. 2017, 117, 6225-6331. doi:10.1021/acs.chemrev.6b00558

3. Novoselov, K. S.; Mishchenko, A.; Carvalho, A.; Castro Neto, A. H. Science 2016, 353, aac9439. doi:10.1126/science.aac9439

4. Yang, X.; Krieger, I.; Lüftner, D.; Weiß, S.; Heepenstrick, T.; Hollerer, M.; Hurdax, P.; Koller, G.; Sokolowski, M.; Puschnig, P.; Ramsey, M. G.; Tautz, F. S.; Soubatch, S. Chem. Commun. 2018, 54, 9039-9042. doi:10.1039/c8cc03334j

5. Gebauer, W.; Langner, A.; Schneider, M.; Sokolowski, M.; Umbach, E. Phys. Rev. B 2004, 69, 155431. doi:10.1103/physrevb.69.155431

6. Chance, R. R.; Prock, A.; Silbey, R. Molecular Fluorescence and Energy Transfer Near Interfaces. In Advances in Chemical Physics; Prigogine, I.; Rice, S. A., Eds.; John Wiley \& Sons, Ltd, 1978; Vol. 37, pp 1-65. doi:10.1002/9780470142561.ch1 
7. Campion, A.; Gallo, A. R.; Harris, C. B.; Robota, H. J.; Whitmore, P. M. Chem. Phys. Lett. 1980, 73, 447-450.

doi:10.1016/0009-2614(80)80692-0

8. Neppl, S.; Bauer, U.; Menzel, D.; Feulner, P.; Shaporenko, A.; Zharnikov, M.; Kao, P.; Allara, D. L. Chem. Phys. Lett. 2007, 447, 227-231. doi:10.1016/j.cplett.2007.09.013

9. Kao, P.; Neppl, S.; Feulner, P.; Allara, D. L.; Zharnikov, M. J. Phys. Chem. C 2010, 114, 13766-13773. doi:10.1021/jp1042816

10. Müller, M.; Le Moal, E.; Scholz, R.; Sokolowski, M. Phys. Rev. B 2011, 83, 241203. doi:10.1103/physrevb.83.241203

11. Luo, Y.; Chen, G.; Zhang, Y.; Zhang, L.; Yu, Y.; Kong, F.; Tian, X.; Zhang, Y.; Shan, C.; Luo, Y.; Yang, J.; Sandoghdar, V.; Dong, Z.; Hou, J. G. Phys. Rev. Lett. 2019, 122, 233901. doi:10.1103/physrevlett.122.233901

12. Imada, H.; Miwa, K.; Imai-Imada, M.; Kawahara, S.; Kimura, K.; Kim, Y. Phys. Rev. Lett. 2017, 119, 013901. doi:10.1103/physrevlett.119.013901

13. Kimura, K.; Miwa, K.; Imada, H.; Imai-Imada, M.; Kawahara, S.; Takeya, J.; Kawai, M.; Galperin, M.; Kim, Y. Nature 2019, 570, 210-213. doi:10.1038/s41586-019-1284-2

14. Watanabe, K.; Taniguchi, T.; Kanda, H. Nat. Mater. 2004, 3, 404-409. doi:10.1038/nmat1134

15. Falin, A.; Cai, Q.; Santos, E. J. G.; Scullion, D.; Qian, D.; Zhang, R.; Yang, Z.; Huang, S.; Watanabe, K.; Taniguchi, T.; Barnett, M. R.; Chen, Y.; Ruoff, R. S.; Li, L. H. Nat. Commun. 2017, 8, 15815. doi:10.1038/ncomms15815

16. Mahvash, F.; Eissa, S.; Bordjiba, T.; Tavares, A. C.; Szkopek, T.; Siaj, M. Sci. Rep. 2017, 7, 42139. doi:10.1038/srep42139

17. Li, L. H.; Cervenka, J.; Watanabe, K.; Taniguchi, T.; Chen, Y. ACS Nano 2014, 8, 1457-1462. doi:10.1021/nn500059s

18. Lee, K. H.; Shin, H.-J.; Lee, J.; Lee, I.-y.; Kim, G.-H.; Choi, J.-Y.; Kim, S.-W. Nano Lett. 2012, 12, 714-718. doi:10.1021/nl203635v

19. Auwärter, W. Surf. Sci. Rep. 2019, 74, 1-95. doi:10.1016/j.surfrep.2018.10.001

20. Paulheim, A.; Marquardt, C.; Aldahhak, H.; Rauls, E.; Schmidt, W. G.; Sokolowski, M. J. Phys. Chem. C 2016, 120, 11926-11937. doi:10.1021/acs.jpcc.6b01956

21. Eisfeld, A.; Marquardt, C.; Paulheim, A.; Sokolowski, M. Phys. Rev. Lett. 2017, 119, 097402. doi:10.1103/physrevlett.119.097402

22. Kerfoot, J.; Korolkov, V. V.; Nizovtsev, A. S.; Jones, R.; Taniguchi, T.; Watanabe, K.; Lesanovsky, I.; Olmos, B.; Besley, N. A.; Besley, E.; Beton, P. H. J. Chem. Phys. 2018, 149, 054701. doi:10.1063/1.5041418

23. Forker, R.; Dienel, T.; Krause, A.; Gruenewald, M.; Meissner, M.; Kirchhuebel, T.; Gröning, O.; Fritz, T. Phys. Rev. B 2016, 93, 165426. doi:10.1103/physrevb.93.165426

24. Joshi, S.; Ecija, D.; Koitz, R.; Iannuzzi, M.; Seitsonen, A. P.; Hutter, J.; Sachdev, H.; Vijayaraghavan, S.; Bischoff, F.; Seufert, K.; Barth, J. V.; Auwärter, W. Nano Lett. 2012, 12, 5821-5828. doi:10.1021/nl303170m

25. Koitz, R.; Seitsonen, A. P.; lannuzzi, M.; Hutter, J. Nanoscale 2013, 5 , 5589. doi:10.1039/c3nr00709j

26. Hite, J. K.; Robinson, Z. R.; Eddy, C. R., Jr.; Feigelson, B. N. ACS Appl. Mater. Interfaces 2015, 7, 15200-15205. doi:10.1021/acsami.5b00723

27. Preobrajenski, A. B.; Vinogradov, A. S.; Mårtensson, N. Surf. Sci. 2005, 582, 21-30. doi:10.1016/j.susc.2005.02.047

28. Lyalin, A.; Nakayama, A.; Uosaki, K.; Taketsugu, T. Top. Catal. 2014, 57, 1032-1041. doi:10.1007/s11244-014-0267-7
29. Lin, S.; Huang, J.; Gao, X. Phys. Chem. Chem. Phys. 2015, 17, 22097-22105. doi:10.1039/c5cp03027g

30. Brülke, C.; Heepenstrick, T.; Humberg, N.; Krieger, I.; Sokolowski, M.; Weiß, S.; Tautz, F. S.; Soubatch, S. J. Phys. Chem. C 2017, 121, 23964-23973. doi:10.1021/acs.jpcc.7b06107

31. Schwarz, M.; Riss, A.; Garnica, M.; Ducke, J.; Deimel, P. S.; Duncan, D. A.; Thakur, P. K.; Lee, T.-L.; Seitsonen, A. P.; Barth, J. V.; Allegretti, F.; Auwärter, W. ACS Nano 2017, 11, 9151-9161. doi:10.1021/acsnano.7b04022

32. Brülke, C.; Heepenstrick, T.; Krieger, I.; Wolff, B.; Yang, X.; Shamsaddinlou, A.; Weiß, S.; Bocquet, F. C.; Tautz, F. S.; Soubatch, S.; Sokolowski, M. Phys. Rev. B 2019, 99, 121404. doi:10.1103/physrevb.99.121404

33. Duhm, S.; Gerlach, A.; Salzmann, I.; Bröker, B.; Johnson, R. L.; Schreiber, F.; Koch, N. Org. Electron. 2008, 9, 111-118. doi:10.1016/j.orgel.2007.10.004

34. Martínez-Galera, A. J.; Gómez-Rodríguez, J. M. J. Phys. Chem. C 2019, 123, 1866-1873. doi:10.1021/acs.jpcc.8b10810

35. Gerlach, A.; Sellner, S.; Schreiber, F.; Koch, N.; Zegenhagen, J. Phys. Rev. B 2007, 75, 045401. doi:10.1103/physrevb.75.045401

36. Ng, M. L.; Preobrajenski, A. B.; Zakharov, A. A.; Vinogradov, A. S.; Krasnikov, S. A.; Cafolla, A. A.; Mårtensson, N. Phys. Rev. B 2010, 81, 115449. doi:10.1103/physrevb.81.115449

37. Koslowski, S.; Rosenblatt, D.; Kabakchiev, A.; Kuhnke, K.; Kern, K.; Schlickum, U. Beilstein J. Nanotechnol. 2017, 8, 1388-1395. doi:10.3762/bjnano.8.140

38. Joshi, S.; Bischoff, F.; Koitz, R.; Ecija, D.; Seufert, K.; Seitsonen, A. P.; Hutter, J.; Diller, K.; Urgel, J. I.; Sachdev, H.; Barth, J. V.; Auwärter, W. ACS Nano 2014, 8, 430-442. doi:10.1021/nn406024m

39. Stallberg, K.; Namgalies, A.; Höfer, U. Phys. Rev. B 2019, 99, 125410. doi:10.1103/physrevb.99.125410

40. Yeshchenko, O. A.; Bondarchuk, I. S.; Losytskyy, M. Y. J. Appl. Phys. 2014, 116, 054309. doi:10.1063/1.4892432

41. Kaiser, R.; Friedrich, M.; Schmitz-Hübsch, T.; Sellam, F.; Kampen, T. U.; Leo, K.; Zahn, D. R. T. Fresenius' J. Anal. Chem. 1999 363, 189-192. doi:10.1007/s002160051169

42. Wagner, V. Phys. Status Solidi A 2001, 188, 1297-1305. doi:10.1002/1521-396x(200112)188:4<1297::aid-pssa1297>3.0.co;2-x

43. Scholz, R.; Kobitski, A. Y.; Kampen, T. U.; Schreiber, M.; Zahn, D. R. T.; Jungnickel, G.; Elstner, M.; Sternberg, M.; Frauenheim, T. Phys. Rev. B 2000, 61, 13659-13669. doi:10.1103/physrevb.61.13659

44. Kilian, L.; Hauschild, A.; Temirov, R.; Soubatch, S.; Schöll, A.; Bendounan, A.; Reinert, F.; Lee, T.-L.; Tautz, F. S.; Sokolowski, M.; Umbach, E. Phys. Rev. Lett. 2008, 100, 136103. doi:10.1103/physrevlett.100.136103

45. Otto, A.; Mrozek, I.; Grabhorn, H.; Akemann, W. J. Phys.: Condens. Matter 1992, 4, 1143-1212. doi:10.1088/0953-8984/4/5/001

46. Schlücker, S. Angew. Chem., Int. Ed. 2014, 53, 4756-4795. doi:10.1002/anie.201205748

47. Cialla, D.; März, A.; Böhme, R.; Theil, F.; Weber, K.; Schmitt, M.; Popp, J. Anal. Bioanal. Chem. 2012, 403, 27-54. doi:10.1007/s00216-011-5631-x

48. García-Vidal, F. J.; Pendry, J. B. Phys. Rev. Lett. 1996, 77, 1163-1166. doi:10.1103/physrevlett.77.1163

49. Xia, M. Coatings 2018, 8, 137. doi:10.3390/coatings8040137

50. Ling, X.; Fang, W.; Lee, Y.-H.; Araujo, P. T.; Zhang, X.; Rodriguez-Nieva, J. F.; Lin, Y.; Zhang, J.; Kong, J.; Dresselhaus, M. S. Nano Lett. 2014, 14, 3033-3040. doi:10.1021/nl404610c 
51. Ehrenreich, H.; Philipp, H. R. Phys. Rev. 1962, 128, 1622-1629. doi:10.1103/physrev.128.1622

52. Babar, S.; Weaver, J. H. Appl. Opt. 2015, 54, 477. doi:10.1364/ao.54.000477

53. Schneider, M. Vibronische und optische Eigenschaften ultradünner organischer Filme am Beispiel PTCDA/Ag(111). Ph.D. Thesis, Universität Würzburg, Würzburg, 2002.

54. Paulheim, A.; Marquardt, C.; Sokolowski, M.; Hochheim, M.; Bredow, T.; Aldahhak, H.; Rauls, E.; Schmidt, W. G. Phys. Chem. Chem. Phys. 2016, 18, 32891-32902. doi:10.1039/c6cp05661j

55. Tenne, D. A.; Park, S.; Kampen, T. U.; Das, A.; Scholz, R.; Zahn, D. R. T. Phys. Rev. B 2000, 61, 14564-14569. doi:10.1103/physrevb.61.14564

56. Hauschild, A.; Temirov, R.; Soubatch, S.; Bauer, O.; Schöll, A.; Cowie, B. C. C.; Lee, T.-L.; Tautz, F. S.; Sokolowski, M. Phys. Rev. B 2010, 81, 125432. doi:10.1103/physrevb.81.125432

57. Hochheim, M.; Paulheim, A.; Sokolowski, M.; Bredow, T. J. Phys. Chem. C 2016, 120, 24240-24249. doi:10.1021/acs.jpcc.6b08540

58. Bauer, O. Surface bonding of a functionalized aromatic molecule: Adsorption configurations of PTCDA on coinage metal surfaces. Ph.D. Thesis, Universität Bonn, Bonn, 2014.

59. Tautz, F. S.; Eremtchenko, M.; Schaefer, J. A.; Sokolowski, M.; Shklover, V.; Umbach, E. Phys. Rev. B 2002, 65, 125405. doi:10.1103/physrevb.65.125405

60. Marquardt, C.; Paulheim, A.; Rohbohm, N.; Merkel, R.; Sokolowski, M. Rev. Sci. Instrum. 2017, 88, 083702. doi:10.1063/1.4997953

61. Gorbachev, R. V.; Riaz, I.; Nair, R. R.; Jalil, R.; Britnell, L.; Belle, B. D.; Hill, E. W.; Novoselov, K. S.; Watanabe, K.; Taniguchi, T.; Geim, A. K.; Blake, P. Small 2011, 7, 465-468. doi:10.1002/smll.201001628

62. Bresnehan, M. S.; Hollander, M. J.; Wetherington, M.; LaBella, M.; Trumbull, K. A.; Cavalero, R.; Snyder, D. W.; Robinson, J. A. ACS Nano 2012, 6, 5234-5241. doi:10.1021/nn300996t

63. Nakhaie, S.; Wofford, J. M.; Schumann, T.; Jahn, U.; Ramsteiner, M.; Hanke, M.; Lopes, J. M. J.; Riechert, H. Appl. Phys. Lett. 2015, 106, 213108. doi:10.1063/1.4921921

64. Xu, Z.; Tian, H.; Khanaki, A.; Zheng, R.; Suja, M.; Liu, J. Sci. Rep. 2017, 7, 43100. doi:10.1038/srep43100

65. Müller, M.; Paulheim, A.; Eisfeld, A.; Sokolowski, M. J. Chem. Phys. 2013, 139, 044302. doi:10.1063/1.4813521

66. Preobrajenski, A. B.; Nesterov, M. A.; Ng, M. L.; Vinogradov, A. S.; Mårtensson, N. Chem. Phys. Lett. 2007, 446, 119-123. doi:10.1016/j.cplett.2007.08.028

67. Schneider, M.; Umbach, E.; Sokolowski, M. Chem. Phys. 2006, 325, 185-192. doi:10.1016/j.chemphys.2005.08.059

68. Wagner, T.; Karacuban, H.; Möller, R. Surf. Sci. 2009, 603, 482-490. doi:10.1016/j.susc.2008.12.007

69. Han, Q.; Shan, H.; Deng, J.; Zhao, A.; Wang, B.; Hou, J. G. Nanoscale 2014, 6, 7934-7939. doi:10.1039/c4nr00017j

70. Wolff, B. Determination of the desorption energy of PTCDA on hBN/Cu(111) by thermal desorption spectroscopy. Master's Thesis, Universität Bonn, Bonn, 2019.

71. Rouillé, G.; Kirchhuebel, T.; Rink, M.; Gruenewald, M.; Kröger, J.; Forker, R.; Fritz, T. Phys. Chem. Chem. Phys. 2015, 17, 30404-30416. doi:10.1039/c5cp03761a

72. Wolff, B. Dynamics of Open Quantum Systems. Master's Thesis, Universität Bonn, Bonn, 2019.

73. Valeur, B. Molecular Fluorescence, 1st ed.; Wiley-VCH: Weinheim, Germany, 2002.
74. Ambrose, W. P.; Basché, T.; Moerner, W. E. J. Chem. Phys. 1991, 95, 7150-7163. doi:10.1063/1.461392

75. Langner, A.; Su, Y.; Sokolowski, M. Phys. Rev. B 2006, 74, 045428. doi:10.1103/physrevb.74.045428

76. Wagner, T.; Bannani, A.; Bobisch, C.; Karacuban, H.; Stöhr, M.; Gabriel, M.; Möller, R. Org. Electron. 2004, 5, 35-43. doi:10.1016/j.orgel.2003.12.001

77. Wagner, T.; Bannani, A.; Bobisch, C.; Karacuban, H.; Möller, R. J. Phys.: Condens. Matter 2007, 19, 056009. doi:10.1088/0953-8984/19/5/056009

78. Forker, R.; Meissner, M.; Fritz, T. Soft Matter 2017, 13, 1748-1758. doi:10.1039/c6sm02688e

79. Barlow, S. M.; Raval, R. Surf. Sci. Rep. 2003, 50, 201-341. doi:10.1016/s0167-5729(03)00015-3

80. Glöckler, K.; Seidel, C.; Soukopp, A.; Sokolowski, M.; Umbach, E.; Böhringer, M.; Berndt, R.; Schneider, W.-D. Surf. Sci. 1998, 405, 1-20. doi:10.1016/s0039-6028(97)00888-1

81. Kilian, L.; Umbach, E.; Sokolowski, M. Surf. Sci. 2006, 600, 2633-2643. doi:10.1016/j.susc.2006.03.049

82. Ogawa, T.; Kuwamoto, K.; Isoda, S.; Kobayashi, T.; Karl, N. Acta Crystallogr., Sect. B: Struct. Sci. 1999, 55, 123-130. doi:10.1107/s0108768198009872

83. Mannsfeld, S.; Toerker, M.; Schmitz-Hübsch, T.; Sellam, F.; Fritz, T.; Leo, K. Org. Electron. 2001, 2, 121-134. doi:10.1016/s1566-1199(01)00018-0

\section{License and Terms}

This is an Open Access article under the terms of the Creative Commons Attribution License (https://creativecommons.org/licenses/by/4.0). Please note that the reuse, redistribution and reproduction in particular requires that the authors and source are credited.

The license is subject to the Beilstein Journal of

Nanotechnology terms and conditions:

(https://www.beilstein-journals.org/bjnano)

The definitive version of this article is the electronic one which can be found at: https://doi.org/10.3762/bjnano.11.149 